\title{
OPEN Identification of pathological transcription in autosomal dominant polycystic kidney disease epithelia
}

\author{
Sebastian Friedrich ${ }^{1,4,5}$, Hannah Müller ${ }^{1,5}$, Caroline Riesterer ${ }^{1,5}$, Hannah Schüller ${ }^{1}$, \\ Katja Friedrich ${ }^{1}$, Carlotta Leonie Wörner ${ }^{1}$, Tilman Busch $^{1}$, Amandine Viau ${ }^{2}$, \\ E. Wolfgang Kuehn ${ }^{1,3}$, Michael Köttgen ${ }^{1,3 凶}$ \& Alexis Hofherr ${ }^{1 \bowtie}$
}

Autosomal dominant polycystic kidney disease (ADPKD) affects more than 12 million people worldwide. Mutations in PKD1 and PKD2 cause cyst formation through unknown mechanisms. To unravel the pathogenic mechanisms in ADPKD, multiple studies have investigated transcriptional mis-regulation in cystic kidneys from patients and mouse models, and numerous dysregulated genes and pathways have been described. Yet, the concordance between studies has been rather limited. Furthermore, the cellular and genetic diversity in cystic kidneys has hampered the identification of mis-expressed genes in kidney epithelial cells with homozygous PKD mutations, which are critical to identify polycystin-dependent pathways. Here we performed transcriptomic analyses of Pkd1and Pkd2-deficient mIMCD3 kidney epithelial cells followed by a meta-analysis to integrate all published ADPKD transcriptomic data sets. Based on the hypothesis that Pkd1 and Pkd2 operate in a common pathway, we first determined transcripts that are differentially regulated by both genes. RNA sequencing of genome-edited ADPKD kidney epithelial cells identified 178 genes that are concordantly regulated by $\mathrm{Pkd} 1$ and $\mathrm{Pkd} 2$. Subsequent integration of existing transcriptomic studies confirmed 31 previously described genes and identified 61 novel genes regulated by Pkd1 and Pkd2. Cluster analyses then linked Pkd1 and Pkd2 to mRNA splicing, specific factors of epithelial mesenchymal transition, post-translational protein modification and epithelial cell differentiation, including CD34, CDH2, CSF2RA, DLX5, HOXC9, PIK3R1, PLCB1 and TLR6. Taken together, this model-based integrative analysis of transcriptomic alterations in ADPKD annotated a conserved core transcriptomic profile and identified novel candidate genes for further experimental studies.

Autosomal dominant polycystic kidney disease (ADPKD) is the most common hereditary nephropathy in humans ${ }^{1}$. Most patients with ADPKD are born healthy, but progressive cystic transformation of both kidneys induces a continuous decline in renal function leading to kidney failure ${ }^{1}$. Mutations in two genes, PKD1 and $P K D 2$, cause $\mathrm{ADPKD}^{2,3}$. The protein products of these genes, polycystin-1 (PC-1) and transient receptor potential channel polycystin-2 (TRPP2) form a receptor-ion channel complex that is essential to establish renal tubular morphology and prevent cystogenesis ${ }^{1}$.

Many cellular signaling pathways have been implicated in the pathogenesis of ADPKD, including Wnt, mTOR, JAK/STAT and Hippo ${ }^{4-8}$. Yet, the precise molecular function of PC-1/TRPP2 and the signaling pathways critical for disease initiation have remained unclear ${ }^{9}$. Unbiased, discovery-based approaches offer unique opportunities to address these key questions of ADPKD pathology. RNA sequencing (RNA-seq) technology has made large scale analysis of gene expression a powerful approach to systematically investigate disease mechanisms ${ }^{10,11}$. Consequently, multiple studies have explored transcription in cystic kidneys from Pkd-deficient mice and

\footnotetext{
${ }^{1}$ Renal Division, Department of Medicine, Medical Center, Faculty of Medicine, University of Freiburg, Hugstetterstrasse 55, 79106 Freiburg, Germany. ${ }^{2}$ Imagine Institute, Laboratory of Hereditary Kidney Diseases, INSERM UMR 1163, Université de Paris, 75015 Paris, France. ${ }^{3} \mathrm{CIBSS}$ - Centre for Integrative Biological Signalling Studies, Freiburg, Germany. ${ }^{4}$ Present address: Department of General Pediatrics, Adolescent Medicine and Neonatology, Center for Pediatrics, Medical Center, Faculty of Medicine, University of Freiburg, Mathildenstrasse 1, 79106 Freiburg, Germany. ${ }^{5}$ These authors contributed equally: Sebastian Friedrich, Hannah Müller and Caroline Riesterer. ${ }^{\circledR}$ email: michael.koettgen@uniklinik-freiburg.de; alexis.hofherr@uniklinik-freiburg.de
} 
Figure 1. Model-guided meta-analysis of ADPKD-responsive transcription identified consistent mis-regulation of 31 known and 61 new genes. Unbiased analysis of Pkd1- and Pkd2-dependent mRNA transcription in genome-edited renal tubular epithelial mIMCD3 ADPKD cells provided rich data with minimal variability. Core Pkd-dependent transcription was delineated by statistical analysis of differential gene expression and exploratory data simplification by principal component analysis. Function-based cluster analysis of identified regulation isolated key pathways caused by loss of Pkd genes. ADPKD disease-relevance was investigated by model-guided meta-analysis of Pkd1- and Pkd2-deficient cells and mice, as well as human PKD1 ${ }^{-1-}$ kidneys. Ultimately, integrative transcriptomics confirmed 31 previously described transcriptional regulations and identified 61 new genes transcriptionally regulated by both, PKD1 and PKD2.

ADPKD patients. These studies have reported a large number of differentially expressed genes in grossly cystic samples $^{12-19}$. However, the concordance between studies for specific gene sets has been low ${ }^{15,19}$. This complex variability is likely to be explained by differences in experimental design, tissue sampling and model system ${ }^{19}$. Hence, transcriptomic profiling of ADPKD has confirmed broad cellular alterations in cystic tissue, but has provided limited novel insights into $\mathrm{PKD}$-dependent tubule-cell-specific factors causing cystic kidney disease.

A key challenge of specific profiling of PKD1- and PKD2-dependent transcription has been the genetic mosaicism of organisms with ADPKD. In ADPKD, cysts arise from individual renal epithelial cells upon somatic loss of heterozygosity for $P K D 1$ or $P K D 2^{20}$. This focal process has been modeled in mice by homozygous Pkd inactivation in kidney tubular epithelial cells. However, in both cases only a small fraction of renal cells harbor the homozygous PKD mutations required to trigger cystogenesis. The majority of cells in polycystic kidneys are still heterozygous for PKD gene mutations-or wildtype in the case of tubule-specific knockout mice. Furthermore, due to secondary processes, such as proliferation of cyst epithelia, inflammation and fibrosis, cystic kidneys show considerable secondary changes in cellular composition ${ }^{21}$. These processes contribute to disease progression, but further confound the transcriptomic profiling of homozygous mutant tubular cells at cyst initiation, a prerequisite to gain insights into PKD gene function.

Here, we investigate cell-autonomous ADPKD-dependent transcriptional changes based on two ground truths: (1) mutations in PKD1 and PKD2 cause ADPKD ${ }^{2,3}$; and (2) renal cystogenesis is initiated in PKD-deficient tubular epithelial cells ${ }^{20}$. First, we characterized transcriptomic profiles of genetically engineered, clonal ADPKD cells in a well-controlled, reductionist approach. RNA-seq of $P k d 1$ - and $P k d 2$-deficient mouse inner medullary collecting duct 3 (mIMCD3) epithelial cells identified a core ADPKD data set of 178 reproducible and concordantly regulated genes. Using this core data set, we next performed a cross-species meta-analysis to integrate the wealth of transcriptomic data from Pkd-deficient animals and human ADPKD kidneys, which confirmed 31 highly consistent, previously described, transcriptionally regulated genes. Notably, we also identified 61 novel genes that are differentially expressed in $P k d 1$ and $P k d 2$ deficiency (Fig. 1). Gene ontology and functional pathway enrichment analyses linked specific factors of epithelial mesenchymal transition (EMT), post-translational protein modification and epithelial cell differentiation to ADPKD pathogenesis. This combination of cellular PKD models with transcriptomic data from cystic kidneys showed the power of integrative transcriptomics to validate candidate targets for in-depth studies of pathogenic mechanisms in ADPKD, including CD34, CDH2, CSF2RA, DLX5, HOXC9, PIK3R1, PLCB1 and TLR6.

\section{Results}

Transcriptional characterization of ADPKD by deep RNA sequencing of Pkd1- and Pkd2-deficient renal tubular cells. Mouse inner medullary collecting duct 3 (mIMCD3) cells represent a well-established, differentiated in vitro model of the distal nephron ${ }^{22}$. mIMCD3 cells are highly differentiated, phenotypically stable and show low heterogeneity, with preserved apico-basolateral polarity, formation of primary cilia, collecting duct-like transport activity and tubule formation in $3 \mathrm{D}$ culture ${ }^{22-25}$. This enables stringent analyses of gene-dependent transcription in a controlled environment, free of systemic variations (Figure S1a) ${ }^{26}$.

Analysis of active gene regulatory networks as major molecular determinants of cell-type identity further confirmed the kidney transcriptional signature of mIMCD3 cells (Figure S1b) ${ }^{27}$. For the targeted evaluation of ADPKD-dependent cellular pathophysiology, we here compared wild-type mIMCD3 cells with constitutive $P k d 1$ - and $P k d 2$-deficient cell lines ${ }^{28}$.

Repeated deep RNA-seq of wild-type, $P k d 1^{-1-}$ and $P k d 2^{-/-}$cells yielded rich and highly consistent transcriptomic profiles (Fig. 2a). Sequencing depth, read distribution between samples, sample-to-sample-distances, dispersion of variance and histogram of p-values verified excellent quality of data (Figure S2a-e).

Collected RNA-seq data confirmed the genomic deletion of $P k d 1$ and $P k d 2$, and showed significant mutual interdependence of $P k d 1$ and $P k d 2$ mRNA expression, indicating a transcriptional $P k d 1 / 2$ feedback loop (Figs. 2b,c, S1c,d). Full gene deletion removed all $P k d 1$ transcripts in mutant cells and was associated with a decrease in $P k d 2$ expression (-31.7\%) (Figs. 2b,c, S1c). Deletion of $P k d 2$, on the other hand, was associated with a significant transcriptional up-regulation of $P k d 1$ mRNA (+54.6\%) (Fig. 2b,c). RNA-seq of $P k d 2^{-1-}$ cells also confirmed that genomic deletion of chromosome 5, bases 104,478,281 - 104,490,878, removed Pkd2 exons 4-9 (of a total 15) and, hence, TRPP2 channel transmembrane domains 2-6 (of a total 6), which generated biochemically TRPP2-deficient cells (Figs. 2c, S1c).

Unbiased statistical integration of Pkd1- and Pkd2-dependent transcription identified a core ADPKD data set of 178 consistently regulated genes $\left(C_{178}\right)$. Mutations in $P K D 1$ and PKD2 cause $\mathrm{ADPKD}^{2,3}$. However, to date, no comprehensive analysis of PKD1- and PKD2-dependent transcription in ADPKD has been reported; several studies have focused on PKD1 and limited data is available on PKD2- 


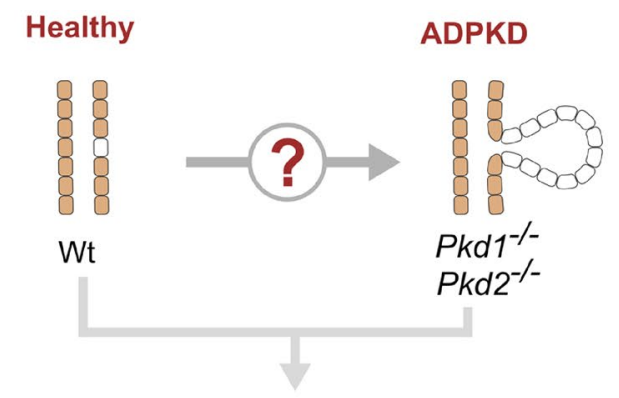

Cell Culture

Model

RNA-seq $=---$

Data Analysis
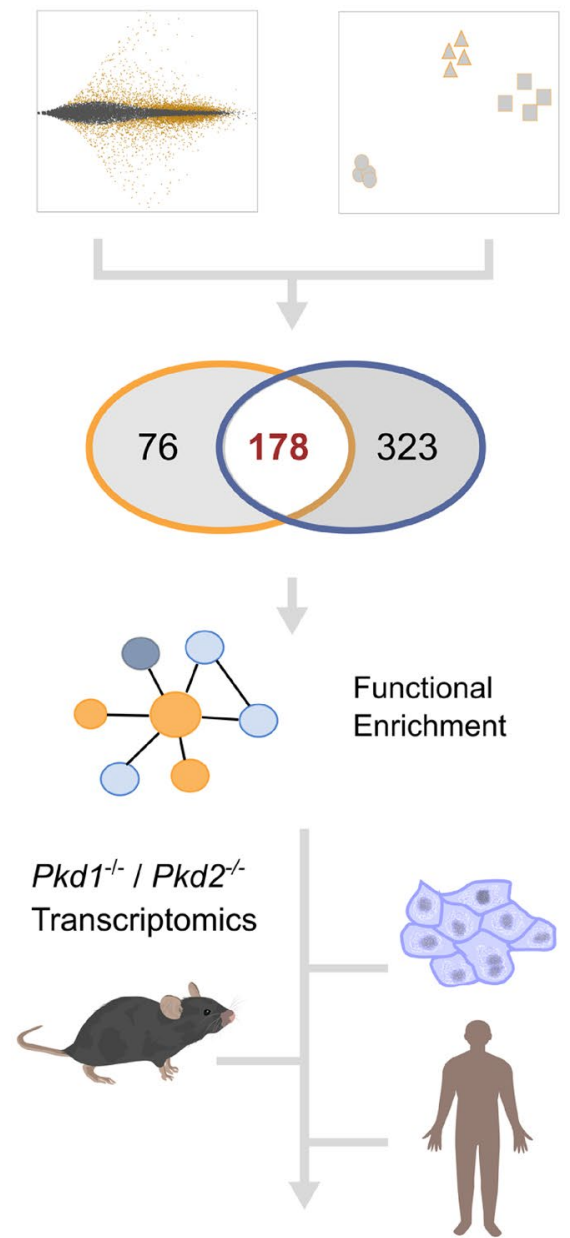

31 Confirmed and $61 \mathrm{New}$ ADPKD Related Genes 
a

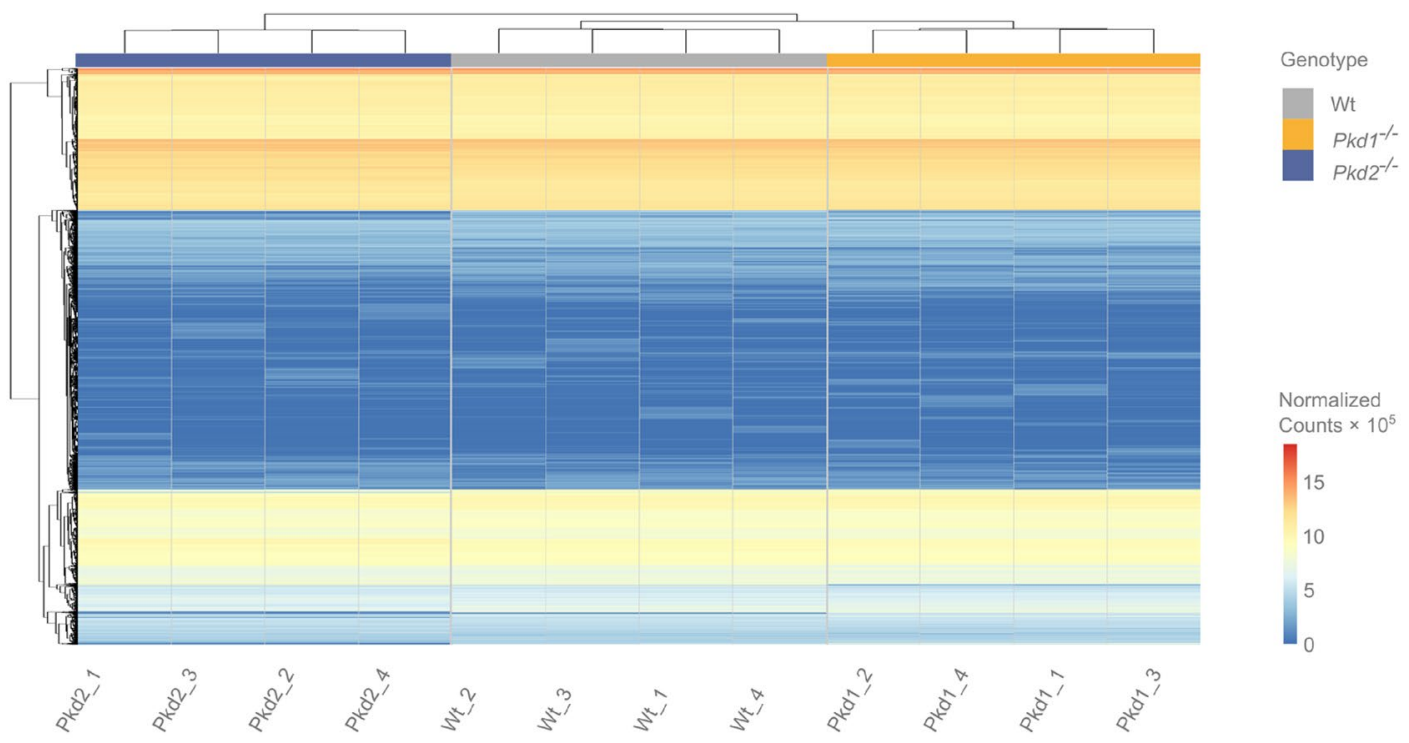

b

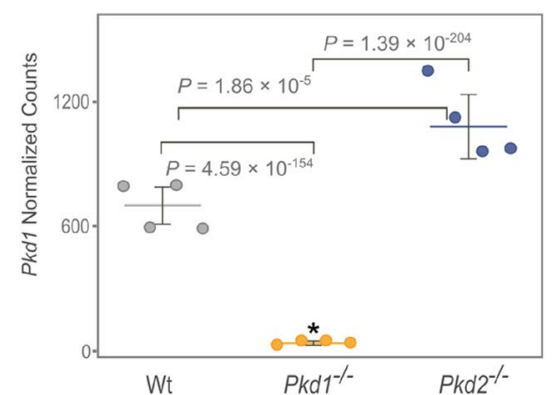

Wt

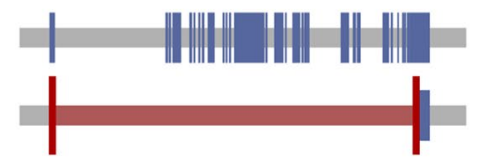

C
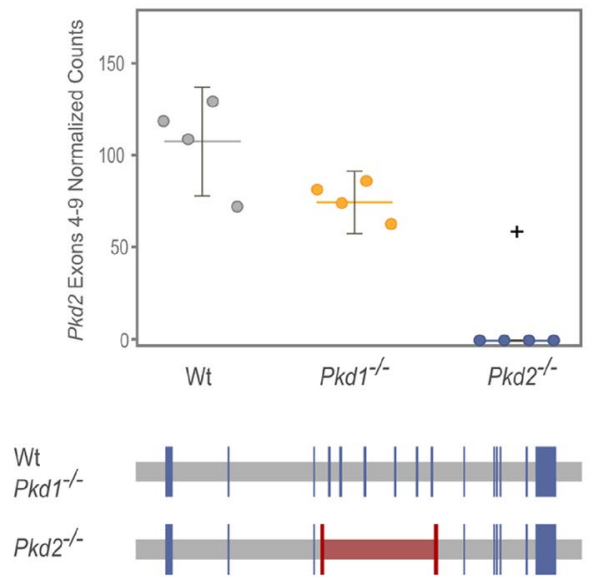

Figure 2. Transcriptomic profiling of genetically engineered ADPKD cells showed significant interdependence of Pkd1 and Pkd2 mRNA expression. (a) Unsupervised hierarchical clustering showed low variability and genotype-dependent grouping of mIMCD3 Wt, $\mathrm{Pkd}^{-/-}$and $\mathrm{Pkd} 2^{-/-}$cells. (b) Pkd1 knockout was generated by a full genomic deletion. Loss of $\mathrm{Pkd} 2$ was associated with a significant transcriptional up-regulation of $\mathrm{Pkd} 1$ mRNA (782.25 [SEM = 97] mean normalized counts in wildtype vs. 1,209 [SEM = 175] in Pkd2 ${ }^{-l-}$ cells). $(\mathbf{c}) \mathrm{Pkd} 2$ knockout was based on genomic deletion of $\mathrm{Pkd} 2$ exons 4-9 and no counts were detected for these exons. + : adjusted $\mathrm{p}$-values for single exons $4-9$ were $<1.75 \times 10^{-51}$ in $\mathrm{Pkd} 2^{-/-}$versus $\mathrm{Wt}$ and $\mathrm{Pkd} 2^{-/-}$versus $\mathrm{Pkd}^{-/-}$cells.

responsive transcription ${ }^{18,19}$. We hypothesized that the integrative analysis of $P K D 1$ - and PKD2-deficiency may substantially improve the high-confidence identification of consistent transcriptional changes in ADPKD pathogenesis.

Technically, the low biological variability of wild-type, $P k d 1^{-/-}$and $P k d 2^{-/-}$mIMCD3 cells provided a sound basis to delineate ADPKD-specific renal tubular transcription (Figs. 2a, S2a-e). This was important, as gene expression is an inherently stochastic process and is known to vary even between cells of the same population ${ }^{29}$. In general, observed variations in gene expression can be decomposed $\mathrm{ss}^{30,31}$ :

$$
\operatorname{Var}(\text { Expr })=\text { Across group variability + Measurement error + Biological variability. }
$$

With group variability being here the variation in gene expression due to the three genotypes under consideration. Measurement error being rather low in next-generation RNA-seq ${ }^{32}$. And biological variability being in our experiments restricted to stochastic cell-to-cell variation; and, therefore, minimal in comparison to tissue or cell type variability in whole organ analyses ${ }^{31}$. Thus, our approach of repeated sampling, high data quality and model simplicity should minimize the confounding effects of measurement error and biological variability, focusing generated data on the primary outcome of interest: the estimated Pkd-dependent difference in gene expression between genotypes. 
Two complementary mathematical methodologies were used to query the raw RNA-seq data for ADPKDspecific gene expression: (1) statistical analysis of differential gene expression (DGE); and (2) unbiased data simplification by principal component analysis (PCA) $)^{33,34}$.

For DGE, statistical comparison of wild-type, $P k d 1^{-1-}$ and $P k d 2^{-/-}$cells identified significant differences in gene expression (Fig. 3a,b). Given that the likelihood of statistically significant results to reflect a true effect is dependent on the respective effect size, we defined DGE positive results by false discovery rate (FDR) $<0.05$, and $\log _{2}$ fold change $\geq|1|^{35,36}$. By this definition, 781 genes in $P k d 1^{-1-}$ and 1150 in $P k d 2^{-1-}$ were identified as differentially expressed vs. wildtype (Fig. 3a,b, Tables S1, S2). From these, the DGE-based Pkd-specific renal tubular gene expression profile was compiled by filtering for concordant changes. Hence, DGE identified 254 (38 up- and 216 down-regulated; $\mathrm{DGE}_{254}$ ) Pkd-specific genes (Fig. 3c and Table S3).

For PCA, raw RNA-seq data was input to unsupervised eigenvector-based multivariate analyses. This dimensionality reduction confirmed the close correlation of individual genotypes (Fig. 3d). Two principal components (PC) explained most sample variance, PC1 with $69 \%$ and PC2 with $29 \%$. The biological meaning of this data structure may be inferred by the two key factors differentiating the evaluated mIMCD3 cell lines: (1) random clonal variation; and (2) Pkd-dependent gene expression. Because PC2 correlated with Pkd1- and Pkd2-deficiency, genes were ranked by PC2 weighting and the top $20 \%\left(\mathrm{n}=501 ; \mathrm{PCA}_{501}\right)$ were selected for comparison with the $\mathrm{DGE}_{254}$ data set (Fig. 3e and Table S4).

Comparison of the independent $\mathrm{DGE}_{254}$ and $\mathrm{PCA}_{501}$ data sets identified 178 distinct genes specifically and concordantly responding to $P k d 1$ - and $P k d 2$-deficiency in mIMCD3 cells (core data set; $\mathrm{CD}_{178}$ ) (Figs. 3f, S3 and Table S5). Crucially, the integration of $P k d 1$ and $P k d 2$ signatures should strongly focus $\mathrm{CD}_{178}$ on transcripts relevant for $\mathrm{ADPKD}$ pathogenesis.

Function-based clustering of the $\mathrm{CD}_{178}$ core ADPKD data set isolated key pathways mediating cyst pathophysiology. Conceptually, $\mathrm{CD}_{178}$ provided an unbiased look at the transcriptional activity of Pkd-deficient kidney cells. To uncover the cellular signaling pathways represented by these core ADPKD genes, we next linked prior biological knowledge to the newly generated $\mathrm{CD}_{178}$ expression data. Analyses were performed on protein and transcript levels.

Protein-protein associations within $\mathrm{CD}_{178}$ were queried using the STRING platform ${ }^{37} .176 \mathrm{CD}_{178}$ genes matched with proteins in the STRING database (Fig. 4a). Notably, STRING network analysis separated 3 highly connected k-Means clusters within $\mathrm{CD}_{178}$ : $(\mathrm{A})$ with 10 proteins $\left(\mathrm{A}_{10}\right)$; $(\mathrm{B})$ with 8 proteins $\left(\mathrm{B}_{8}\right)$; and $(\mathrm{C})$ with 7 proteins $\left(\mathrm{C}_{7}\right.$; total $\left.=\mathrm{ABC}_{25}\right)$ (Fig. 4a). To interpret the identified protein interactions we performed unguided as well as cluster-guided gene set enrichment analysis (GSEA) using a FDR $<0.05^{38}$.

Unguided GSEA correlated previously defined hallmark gene sets to $\mathrm{CD}_{178}$; with Epithelial Mesenchymal Transition (EMT) being the most significantly correlated hallmark gene set (FDR $=1.79 \times 10^{-11}$ ), supporting the previously described role of EMT in ADPKD cystogenesis (Fig. 4b) ${ }^{16,39}$. Given the smaller size of $\mathrm{ABC}_{25}$ and, hence, smaller possibility space, cluster-guided GSEA was complemented with gene ontology (GO) analysis, transcription factor target analysis and evaluation of the Kyoto Encyclopedia of Genes and Genomes (KEGG)

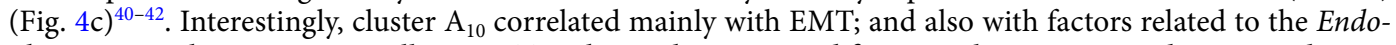
plasmic Reticulum Lumen as well as Post Translational Protein Modification. Cluster $\mathrm{B}_{8}$ instead comprised genes related to Epithelial Cell Differentiation, Animal Organ Morphogenesis and Sequence Specific DNA Binding, suggesting a role in tissue formation. And cluster $\mathrm{C}_{7}$ seemed exclusively related to phosphorylation-dependent cellular signaling (Fig. 4c). For full GSEA results, see Table S6.

The transcriptional regulation of $\mathrm{CD}_{178}$ was probed by transcription factor enrichment analysis using the ShinyGO and GeneSetDB meta-databases ${ }^{41,42}$. Seven transcription factors were identified to be significantly enriched in the regulation of $\mathrm{CD}_{178}\left(\mathrm{FDR}<0.05 ; \mathrm{Ar}, \mathrm{Cebpd}\right.$, Esr1, Ets2, Gli1, Tp53 and Rbpj; $\left.\mathrm{TF}_{7}\right)$, controlling at least 17 of the 178 genes in $\mathrm{CD}_{178}$ (Fig. $4 \mathrm{~d}, \mathrm{e}$ ). Targeted expression analysis of $\mathrm{TF}_{7}$ demonstrated for Esr1 a significant down-regulation in $P k d 1^{-/-}$and $P k d 2^{-/-}$cells $\left(\log _{2}\right.$ fold change $=-1.04, \mathrm{FDR}=0.003$ and $\log _{2}$ fold change $=-1.54, \mathrm{FDR}=1.78 \times 10^{-6}$, respectively); none of the other $6 \mathrm{TF}_{7}$ factors showed consistent, $P k d 1$ - and $P k d 2$-dependent differences in gene expression (Table S7). Because the PC-1/TRPP2 receptor-ion channel structure makes a direct control of cellular transcription unlikely, it is intriguing to speculate that non-transcriptional polycystin signaling regulates $\mathrm{TF}_{7}$ activity, which in turn may control PKD-dependent gene expression in kidney tubular epithelial cells ${ }^{43}$. $\mathrm{Ca}^{2+}$-regulation and phosphorylation-dependence, for example, have been described for the zinc-finger protein GLI1 and the cellular tumor antigen $\mathrm{p} 53^{44,45} \cdot \mathrm{TF}_{7}$ transcription factors may, hence, provide a mechanistic link from PC-1/TRPP2 channel activity to down-stream regulation of cellular gene expression.

Another important contributor to both protein-protein associations and control of gene expression levels is alternative splicing of pre-mRNAs ${ }^{46}$. To date, alternative splicing has not been reported in ADPKD. Analysis of differential exon usage in our wild-type, $P k d 1^{-1-}$ and $P k d 2^{-/-}$RNA-seq data identified 176 Pkd-responsive exons in 107 distinct genes (Fig. 5a,b). Within $\mathrm{CD}_{178}$, three genes (Pik3r1, Bex4 and Slc16a3) showed significant differences in exon usage (Fig. 5c). Similarly, TF $_{7}$ Rbpj exon usage seemed to be Pkd-dependent (Fig. 5d). Notably, $P k d 1$ exons $11,15,34$ and 46 were differentially spliced in $P k d 2$-deficient cells (Fig. 5e,f). This indicated that functional TRPP2 is required for both, the amount and the splice variant of $P k d 1$ gene expression (Figures S1c, $5 e, f)$. Although direct derivation of biologically meaningful insights from these data is challenging, it reinforced the intimate mutual regulation of PKD genes and PC-1/TRPP2 proteins.

Furthermore, integration of protein association- and transcription factor-derived networks delineated putative Pkd-dependent regulatory cascades in mIMCD3 cells: $5 \mathrm{ABC}_{25}$ genes (Cd34, Spp1, Cdh2, C3 and Mmp2) were linked to 6 of the $\mathrm{TF}_{7}$ transcription factors, thus, further connecting phenotypic ADPKD pathogenesis to the unknown cellular functions of PKD1 and PKD2 (Fig. 4e). 
a

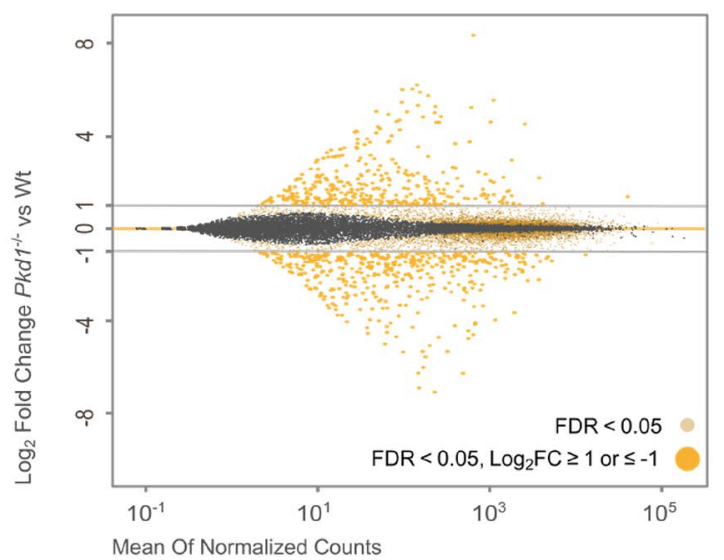

b

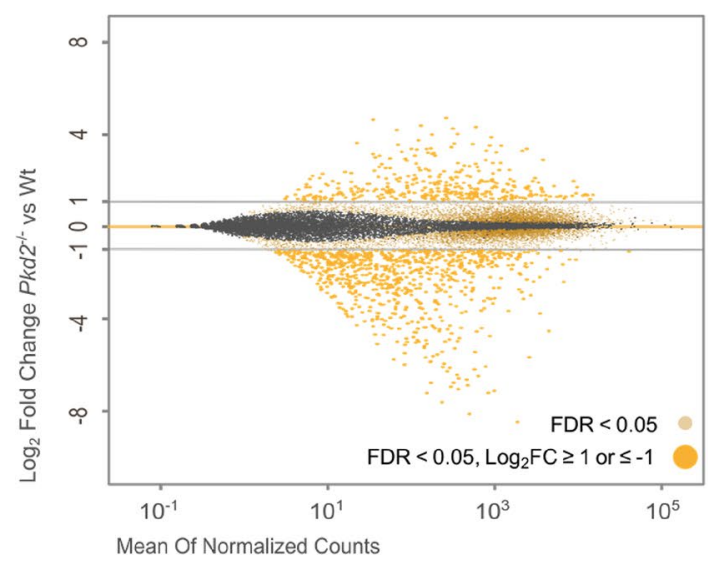

d

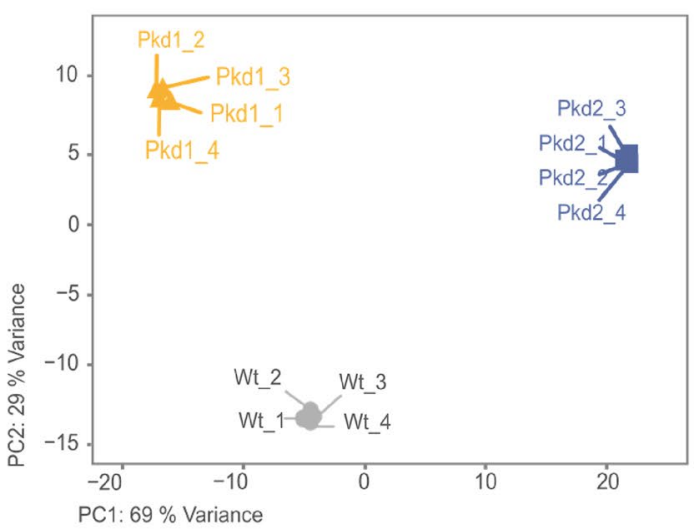

f

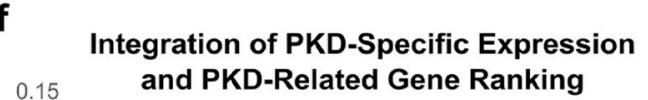

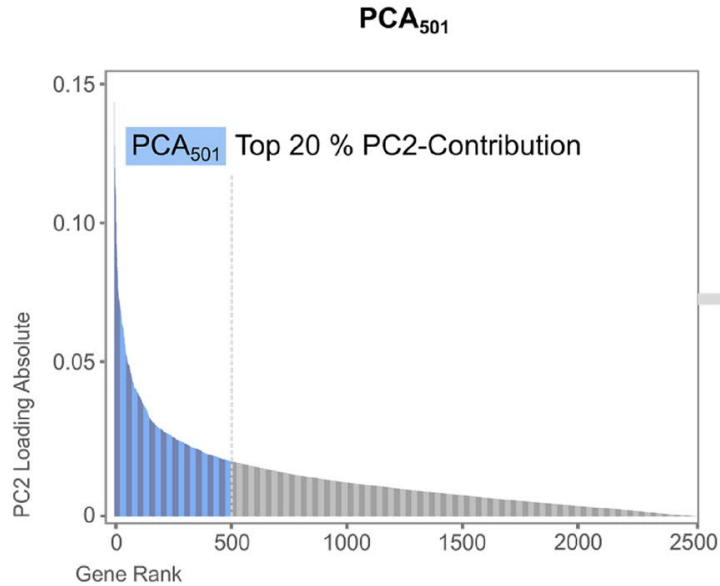

Gene Rank

\section{$\mathrm{DGE}_{254}$}
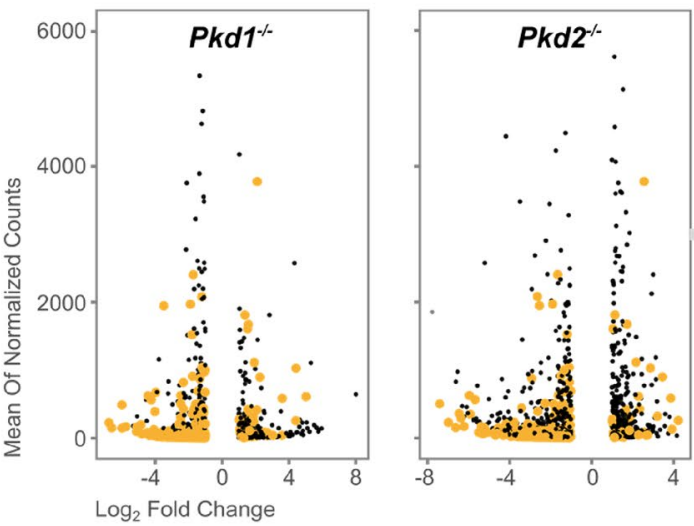

e

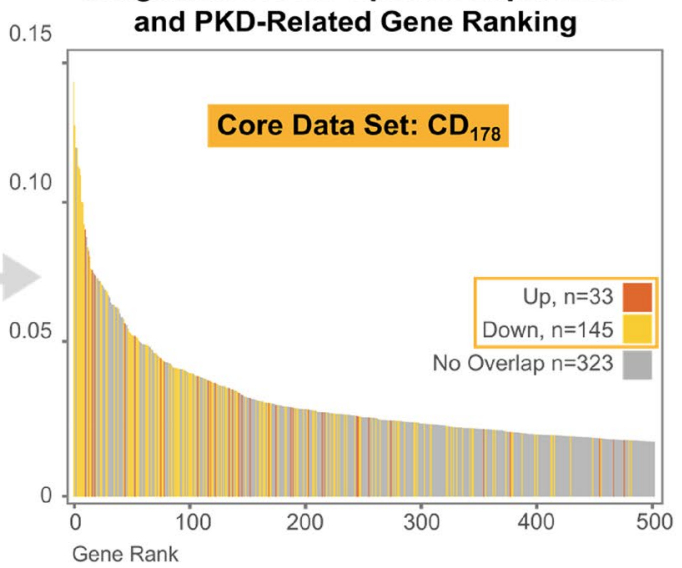

Figure 3. Complementary mathematical analysis of Pkd1- and Pkd2-responsive transcripts identified a core ADPKD data set of 178 consistently regulated genes $\left(\mathrm{CD}_{178}\right)$. To identify genes involved in ADPKD pathogenesis, two independent mathematical approaches were used: (1) statistical analysis of differential gene expression (DGE); and (2) unbiased data simplification by principal component analysis (PCA). (a) and (b) Applying RNA-seq on Pkd1- and Pkd2-deficient mIMCD3 cells revealed a total of 791 differentially expressed genes in $\mathrm{Pkd1}^{-/-}$and 1250 in Pkd2 ${ }^{-/-}$cells (large, dark-yellow dots: $\mathrm{FDR}<0.05, \log _{2}$ fold change $\geq|1|$ ). $\operatorname{In~Pkd1~}^{-/-}$cells, $>54 \%$ (429 genes) and in $\mathrm{Pkd}_{2}{ }^{-/-}$cells, $>63 \%$ (790 genes) were downregulated. (c) Filtering DGE of $\mathrm{Pkd}^{-/-}$and $\mathrm{Pkd}^{-/-}$for concordant changes provided 254 genes $\left(\mathrm{DGE}_{254}\right.$ ), of which 38 were up- and 216 downregulated genes (orange dots). (d) 10\% most variant genes were input for PCA ( $\mathrm{n}=2,506$, with reads obtained for 25,062 genes). Principal component 1 (PC1) mainly accounted for non-Pkdrelated differences between wildtype, Pkd1 and Pkd2 cells. PC2 discriminated Pkd1 $1^{-/-}$and Pkd2 ${ }^{-/}$from wildtype, accounting for $29 \%$ of total variance. (e) Since PC2 correlated with Pkd1- and Pkd2-deficiency, genes were ranked according to their PC2 contribution, and the top 20\% ( $\mathrm{n}=501$, marked in blue) were selected for further data analysis $\left(\mathrm{PCA}_{501}\right)$. (f) Comparison of $\mathrm{DGE}_{254}$ and $\mathrm{PCA}_{501}$ identified 178 candidate genes specifically responding to Pkd1- and Pkd2-deficiency in mIMCD3 cells (core data set; $\mathrm{CD}_{178}$ ). 


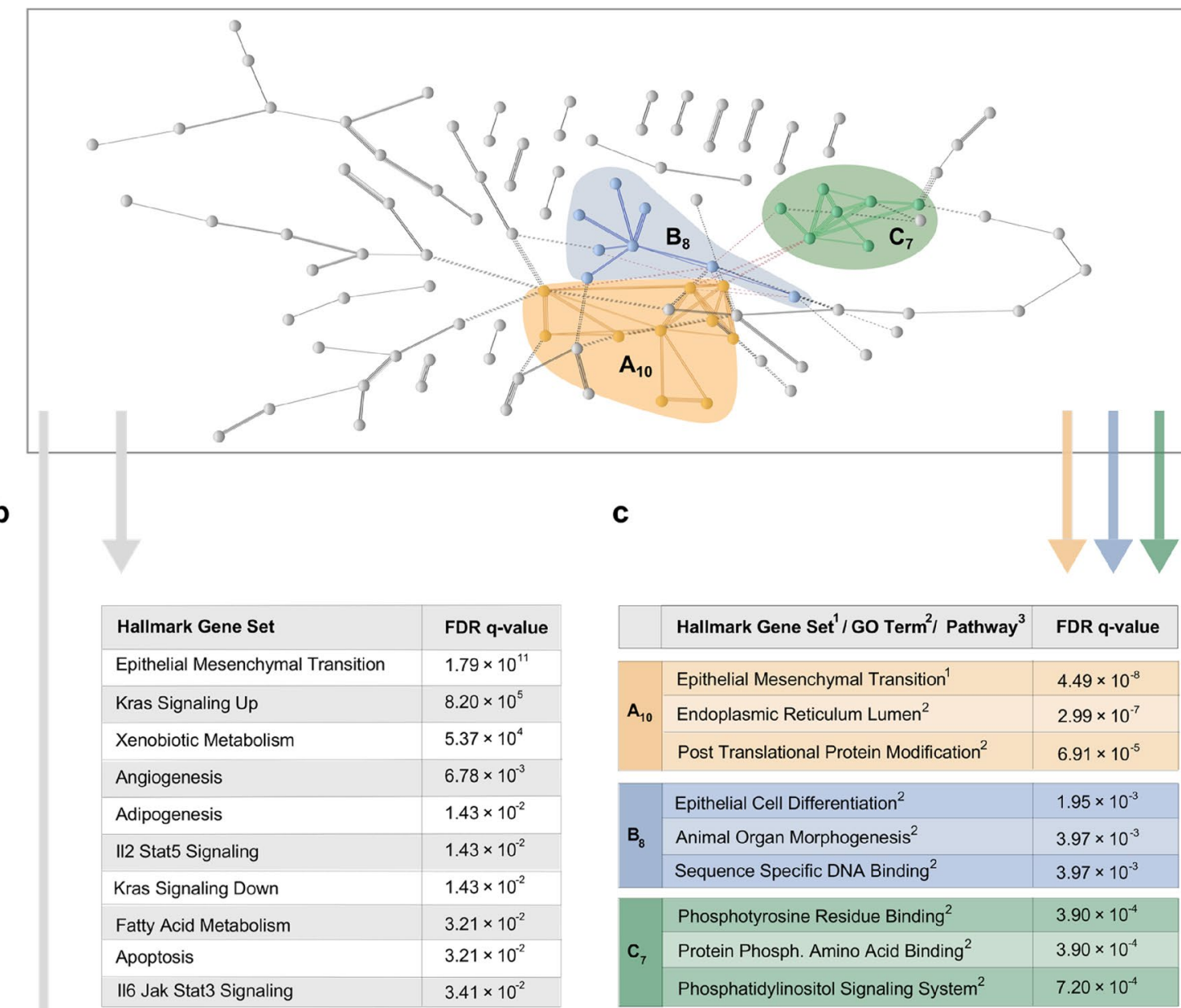

d

Identification of Transcriptional Factors Regulating Genes in the Core Data Set
Network of Transcriptional Factors

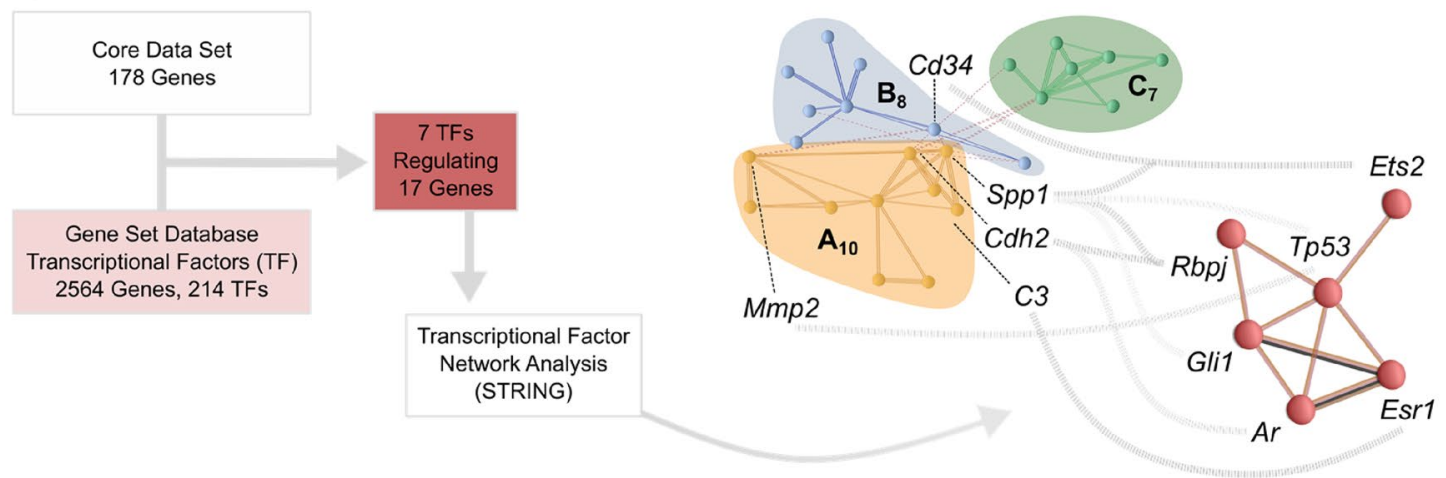

Figure 4. Function-based mapping of $\mathrm{CD}_{178}$ identified $\mathrm{ADPKD}$-related protein networks. (a) $\mathrm{CD}_{178}$ was used as input for STRING network analysis ${ }^{37}$. No annotations were available for 2610203C20Rik and 6330403L08Rik. Application of the k-means algorithm to $C_{178}$ revealed 3 distinct clusters: $A$ with 10 proteins $\left(A_{10}\right)$; B with 8 proteins $\left(B_{8}\right)$; and $C$ with 7 proteins $\left(\mathrm{C}_{7}\right.$; total $\left.=\mathrm{ABC}_{25}\right)$. (b) and (c) to facilitate interpretation of the observed clusters, gene set enrichment analysis (GSEA) was applied to both $\mathrm{CD}_{178}$ and $\mathrm{ABC}_{25}(\mathrm{FDR}<0.05)$. $\mathrm{ABC}_{25}$ genes were subjected to an extended analysis, comprising hallmark gene sets, gene ontology $(\mathrm{GO})$ analysis, transcription factor target analysis and evaluation of the Kyoto Encyclopedia of Genes and Genomes (KEGG) ${ }^{42,96-98}$. See Table S7 for full results. (d) To elucidate $\mathrm{CD}_{178}$ transcriptional regulation, ShinyGO and GeneSetDB meta-databases were queried $(\mathrm{FDR}<0.05)^{41,42}$. This revealed a total of $17 \mathrm{CD}_{178}$ genes regulated by 7 trancriptional factors $\left(\mathrm{TF}_{7}\right)$. (e) 6 of $7 \mathrm{TF}_{7}$ were shown to form a k-means based cluster. Also, $\mathrm{ABC}_{25}$ identified several regulatory cascades: 5 $\mathrm{ABC}_{25}$ genes (Cd34, Spp1, Cdh2, C3 and Mmp2) were linked to 6 of the TF 7 (Ar, Esr1, Ets, Gli1, Rbpj, Tp53). Spp1 and Cdh2 were regulated by more than one $\mathrm{TF}_{7}$, whereas Ets2, Rbpj and $\mathrm{Tp} 53$ regulated more than one $\mathrm{ABC}_{25}$ gene. 
a

Differential Exon Usage

C

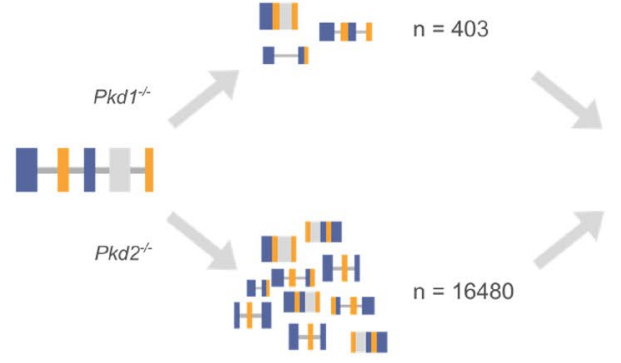

C

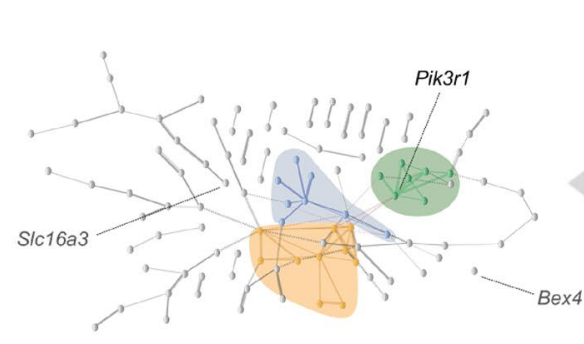

e

\section{$P k d 1$ was Differentially Spliced in $P k d 2^{-/-}$}

b

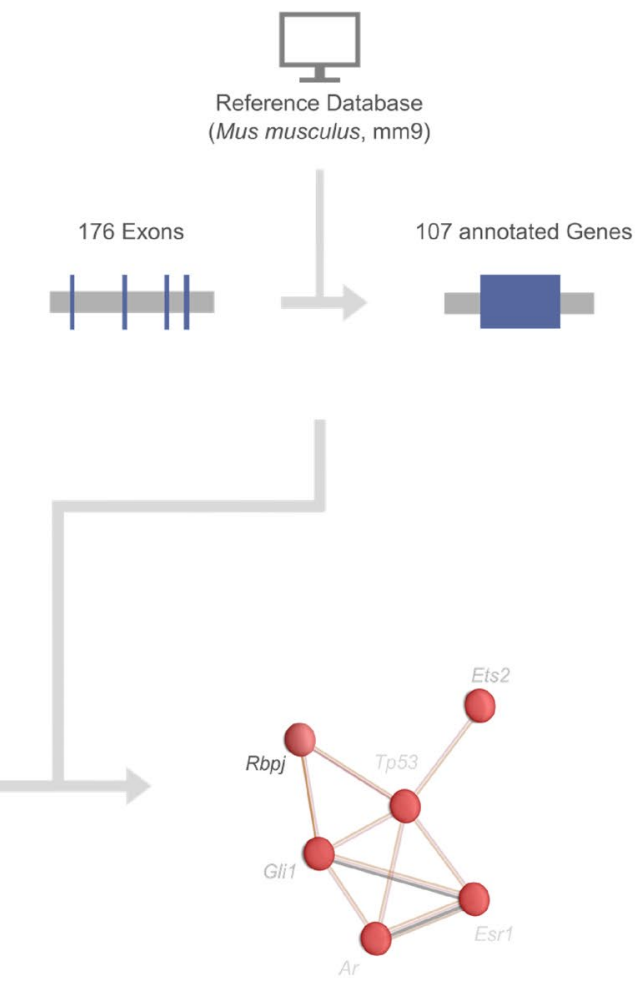

$\mathbf{f}$

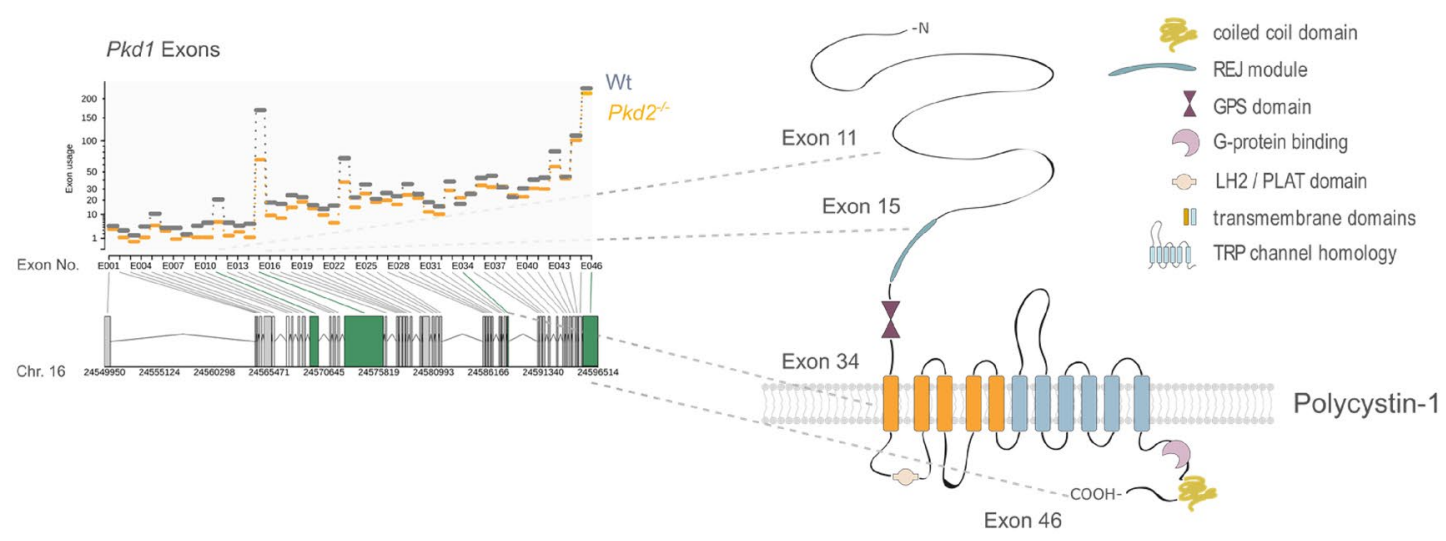

Figure 5. Loss of $P k d 1$ and $P k d 2$ caused significant alteration in exon usage, including differential splicing of Pkd1. (a) Differential exon usage in $\mathrm{Pkd1}^{-/-}$and $\mathrm{Pkd} 2^{-/-}$cells was assessed using DEXSeq ${ }^{99,100}$. Surprisingly, the number of transcripts with differential exon usage compared to wildtype was $>70$-fold higher in Pkd2deficient cells than in $\mathrm{Pkd1}^{-/-}$cells $(\mathrm{n}=16,480$ vs. $\mathrm{n}=403)$. (b) 176 differentially used exons overlapped between both genotypes. Identified exons mapped to 107 annotated genes and 39 genomic loci without annotation. 107 characterized genes are thus differentially spliced in $\mathrm{Pkd}^{-/-}$and $\mathrm{Pkd}^{-/-}$cells. (c) Within the $\mathrm{CD}_{178}$ core set of genes with Pkd-dependent mRNA expression, Pik3r1, Bex4 and Slc16a3 showed additional Pkd-dependent alterations in exon usage. Interestingly, Pik3r1 was associated with protein phosphorylation in functional cluster $\mathrm{C}_{7}$ (Fig. 4c). (d) $\mathrm{TF}_{7}$ transcription factor Rbpj showed differential splicing in $\mathrm{Pkd1}^{-1-}$ and $\mathrm{Pkd}^{-/-}$cells. Rbpj activity had been linked to the expression of $4 \mathrm{CD}_{178}$ genes: Cdh2, Glul, Mt2 and Spp1 (Fig. 4e). (e) and (f) further corroborated the functional interdependence of PKD1 and PKD2, Pkd1 exons 11, 15, 34 and 46 showed reduced usage in $\mathrm{Pkd} 2^{-/-}$cells. 4 domains of Polycystin-1 (PC-1) were affected: exons 11 and 15 project to the long extracellular N-terminus of PC-1, with exon 15 being part of the receptor of egg jelly module; exon 34 contributes to formation of the transmembrane domains; and exon 46 forms part of the intracellular C-terminus. 
Combining published and newly generated data on PKD1 and PKD2 deficiency generated a cross-species transcriptional profile of ADPKD and identified 9 novel targets for future development. The genetic contrast of wild-type and specifically engineered mutant renal tubular epithelial cells provided the opportunity to assess the intricacies of Pkd-dependent transcriptional regulation in simplified cellular isolation. To weigh the relative importance of newly identified $\mathrm{CD}_{178}$ pathways in the complex ADPKD in vivo pathophysiology, we next expanded our investigation to the in vitro in vivo correlation of $\mathrm{CD}_{178}$-derived pathways.

Renal expression of $\mathrm{CD}_{178}$ genes was analyzed by cell type ${ }^{47}$. As expected by the mIMCD3 data source, all $\mathrm{CD}_{178}$ genes were consistently expressed along the nephron without particular cortical or juxtamedullary enrichment (Figure S4a-c). Within the nephron, parietal cells $(>60 \%)$, collecting duct $(>40 \%)$ and medullary pelvis epithelium $(>50 \%)$ show the highest expression of $\mathrm{CD}_{178}$ genes (Figure $44 \mathrm{a}-\mathrm{c}$ ).

To evaluate the relevance of the newly identified $\mathrm{CD}_{178}$ genes for ADPKD pathophysiology we performed a comprehensive cross-species meta-analysis of PKD-dependent RNA expression. At the time of analysis, data were available from six studies evaluating PKD1-dependent transcription and one evaluating loss of $P k d 2$ : one

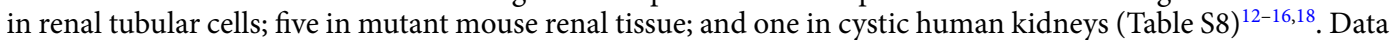
from non-orthologous models of ADPKD were not included in the analysis. For internal validation, we furthermore performed RNA-seq of whole kidneys from littermate control and adult-onset conditional $P k d 1^{-1-}$ mice

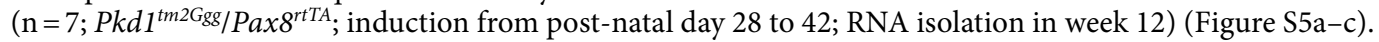

Pooled analysis of aggregated data showed that expression of $117 \mathrm{CD}_{178}$ genes had been described previously as $P K D$-dependent (Figure S6a). Of those, 31 replicated in $\geq 3$ independent transcriptional profiles $\left(\operatorname{Rep}_{31}\right)$, with six genes (ALDH2, APLN, GAL3ST1, HS6ST2, PANK1 and PCSK9) showing concordant regulation across studies, substantiating their pathophysiological relevance in ADPKD (Figs. 6a,b, S6b,c). 61 PKD1/2-dependent genes in $\mathrm{CD}_{178}$, on the other hand, had not been associated with ADPKD before $\left(\mathrm{New}_{61}\right)$ (Fig. 6a,c).

To expand our mechanistic understanding of $\mathrm{New}_{61}$ in PC-1/TRPP2 signaling and identify novel targets for development in ADPKD, we probed the pathophysiological relevance of respective genes by applied bayesian inference ${ }^{48}$ : (1) Through identification, $\mathrm{New}_{61}$ genes were selected to show significant concordant changes $\left(\log _{2}\right.$ fold change $\geq|1|$ ) in $P k d 1$ - and $P k d 2$-deficient mIMCD3 cells (Fig. 6c). (2) Analysis of renal cell type-specific mRNA profiles and comparison to Rep ${ }_{31}$ expression validated disease relevant tissue expression for $49 \mathrm{New}_{61}$ genes (Figure S4) ${ }^{49}$. And importantly, (3) qualitative $\mathrm{Rep}_{31}$-based pathway mapping showed a significant functional correlation of $\mathrm{Rep}_{31}$ and $\mathrm{New}_{61}$. This cross-correlation was most prominent in the $\mathrm{ABC}_{25}$ cluster, which directly linked 6 replicated (C3, FSTL1, PCOLCE, PCSK9, SPP1 and ZFP37) and 8 newly identified (CD34, CDH2, CSF2RA, DLX5, HOXC9, PIK3R1, PLCB1 and TLR6) genes (Fig. 6d). It is, hence, obvious to hypothesize about the relative contributions of $\mathrm{ABC}_{25}$ genes and pathways in loss-of-PKD-dependent cystogenesis and tissue transformation. FSTL1, for example, has been linked to kidney fibrosis and TLR6 to proinflammatory signaling pathways in tubulointerstitial disease $e^{50,51}$.

Taken together, our unbiased, model-guided meta-analysis of PKD-responsive transcription clarified conserved cellular signaling pathways in cystogenesis and identified 8 novel high confidence targets for in-depth analysis in ADPKD.

\section{Discussion}

Transcriptomics has emerged as a powerful systems approach for exploring cellular responses to disease-causing mutations ${ }^{52}$. Some discoveries have already translated to the development of novel preventative and therapeutic strategies, including enzyme replacement therapies in deficiency disorders, antisense oligonucleotide therapies for spinal muscle atrophy and channel modulators for gain-of-function channelopathies ${ }^{53-55}$. However, extracting medically relevant changes in mRNA expression from large transcriptional profiles is a challenging task. The indirect link between mRNA abundance and respective protein activity is a key biological limitation ${ }^{56}$. Experimental setup, choice of model system or stage of disease generally limit validity ${ }^{57}$. The limited concordance of previous ADPKD transcriptomic analysis, particular from animal models clearly illustrate these challenges ${ }^{15,19}$. Here, we applied a genetics-guided data science approach to address these constraints and to reveal core genes and transcriptional pathways that have a high likelihood to promote ADPKD pathology. Methodologically, the kidney epithelium-specific transcriptome of ADPKD was profiled by comparing wild-type mIMCD3 cells with one clonal $P k d 1^{-1-}$ and one $P k d 2^{-/-}$mIMCD3 cell line. Unbiased differential gene expression (DGE) and principal component analysis (PCA) were used to minimize the probability of erroneous inference and provide a robust ADPKD reference profile for more complex disease models.

Data simplification by PCA is designed to reduce data dimensions and facilitate approximation of data. Data dimensionality in PCA is mathematically reduced by transforming a number of correlated variables into uncorrelated principal components (PCs). In our experiment, PC1 explained 69\% of sample variance and PC2 29\%. PC1 is most likely reflective of non-genetic clonal variation due to epigenetics and stochastic gene expression as well as divergent $P k d 1 / 2$ signaling in mIMCD 3 cells (Figs. 3d, S3a-e). Furthermore, the contribution of PC1 to total sample variance is in line with previous analyses of clonal variation in various cell types ${ }^{58,59}$. PC2, on the other hand, better correlated with $P k d 1 / 2$ gene expression (Fig. 3d). The significant overlap of differentially expressed genes $\left(\mathrm{DGE}_{251}\right)$ and PC2-based $\mathrm{PCA}_{501}$ in the $P k d 1 / 2$-dependent $\mathrm{CD}_{178}$ core data provided critical evidence for this argument (Fig. 3f).

The potential biological relevance of $\mathrm{CD}_{178}$ in vivo was subsequently validated by meta-analysis of independent ADPKD transcriptomes, including new data from a $\mathrm{Pkd1}^{-/-}$mouse model (Fig. 6a-d). Putative confounding factors of this approach remain our incomplete understanding of the relevant ADPKD phenotype in vitro as well as missing or biased information in reference biobanks ${ }^{60}$. Still, available data was sufficient to identify the $P K D 1 / 2>\mathrm{TF}_{7}>\mathrm{ABC}_{25}$ hierarchical regulatory network, which may comprise core pathways amplifying 
a

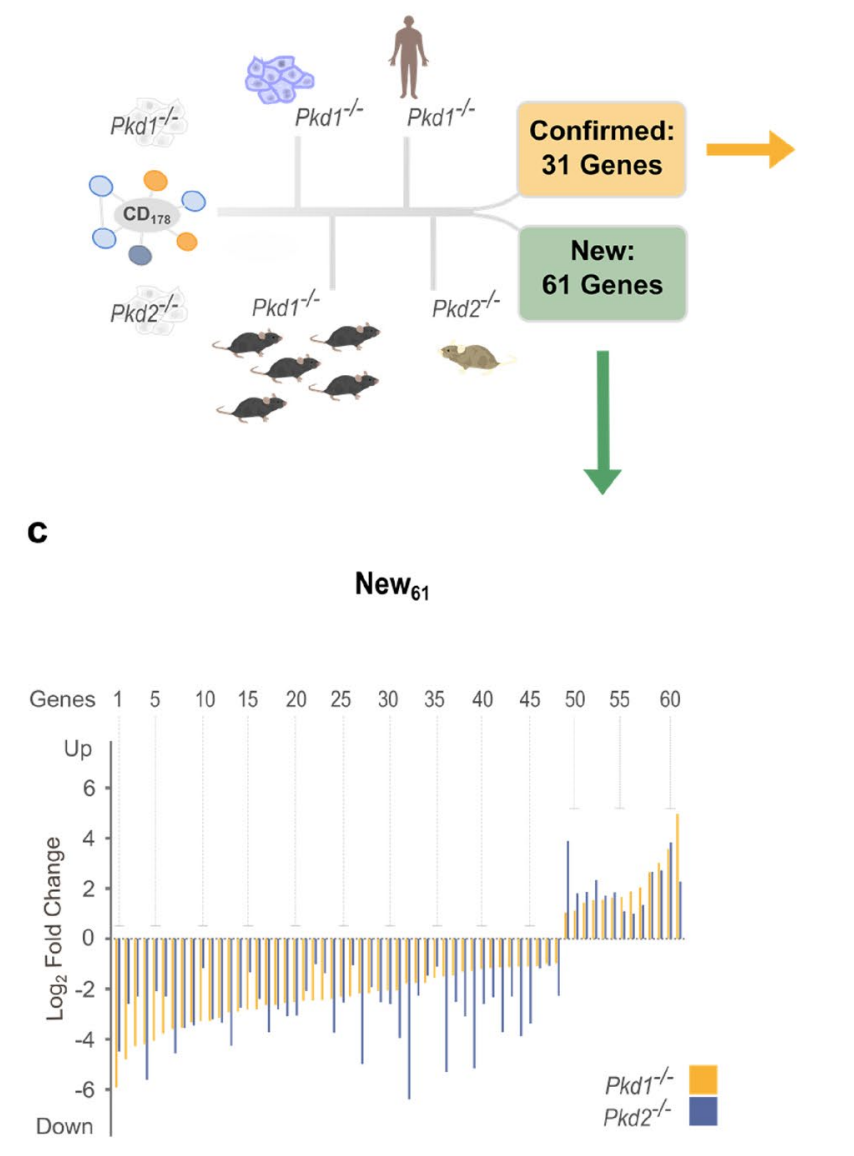

C

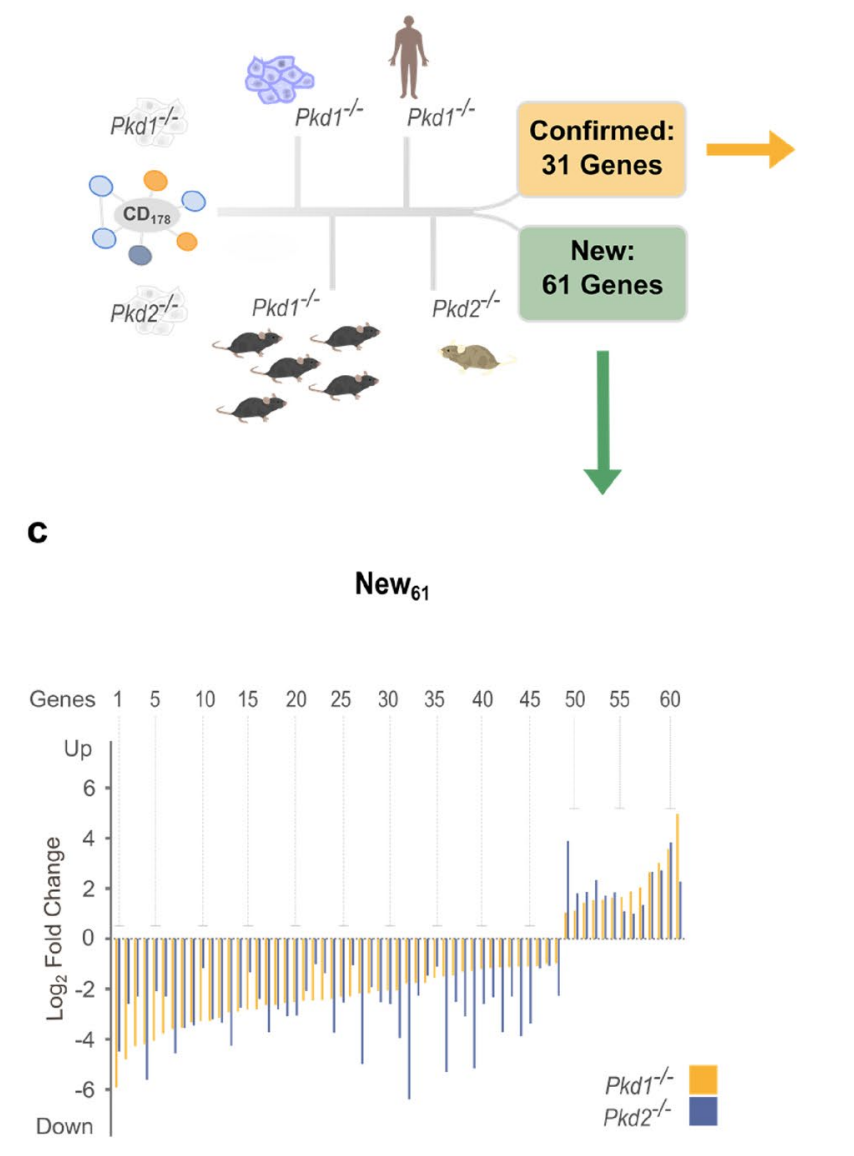

b
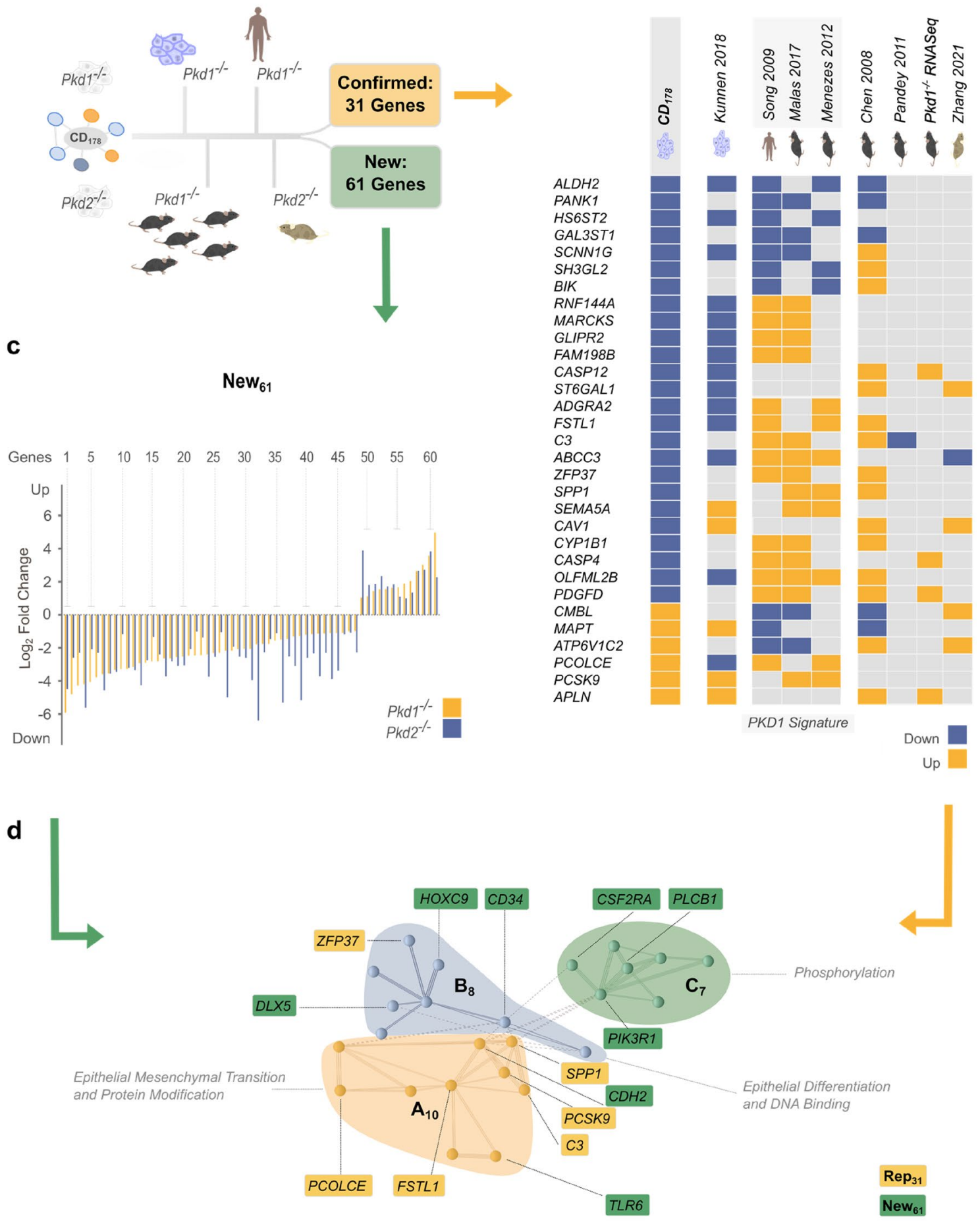

Figure 6. Cross-species meta-analysis of independent ADPKD transcriptomic profiles. (a) 7 independent Pkd1 $1^{-/-}$ transcriptomic studies were chosen for a meta-analysis approach, comprising cell culture, mouse tissue as well as human kidney samples ${ }^{12-16,18} .6$ studies had been previously published, 3 in another meta-analysis ${ }^{15} .1$ mouse model ( $\mathrm{Pkd}^{-l-}$ RNA-seq) was established for independent confirmation (Figure S5). 31 genes were identified in the core data set and at least 3 other studies. 61 genes are reported in the core data set only. (b) Of 31 confirmed genes Rep , $_{31}$ 2 showed concordant upregulation, 4 downregulation. Interestingly, 10 genes are concordantly regulated in cell culture models with discordant regulation in animal/human models. (c) Complementing published data sets, $\mathrm{CD}_{178}$ combined regulation by $\mathrm{Pkd}^{-/-}$and $\mathrm{Pkd}_{2}{ }^{-/-}$. A total of 61 genes appeared in $\mathrm{CD}_{178}$ only $\left(\mathrm{New}_{61}\right)$. (d) Combining the cross-species meta-analysis with function-based mapping and gene enrichment analysis of $\mathrm{ABC}_{25}$ highlighted 8 yet unreported and 6 confirmed genes. Both, confirmed and new genes were represented in clusters $A_{10}$ and $B_{8}$. Interestingly, none of the $\mathrm{Rep}_{31}$ but 3 of $\mathrm{New}_{61}$ genes were represented in functional cluster $\mathrm{C}_{7}$. Thus, combining $\mathrm{Pkd}^{-/-}$and $\mathrm{Pkd}^{-/-}$identified new targets for in-depth analysis of ADPKD pathogenesis. 
transient polycystin-dependent ion flux into stable cellular differentiation and renal tubular structure (Figs. 4e, $6 \mathrm{~d}$ ). Derived from the largest volume of ADPKD mRNA expression data available, the $P K D 1 / 2>\mathrm{TF}_{7}>\mathrm{ABC}_{25}$ signaling cascade - and in particular the 6 replicated and 8 newly identified genes-generated novel, testable hypotheses that may broaden our understanding of PKD-dependent tissue homeostasis in health and disease.

For example, sex has been described as significant determinant of ADPKD disease severity ${ }^{61}$. Men with ADPKD often have worse renal cystic disease but less severe liver cystic disease ${ }^{62}$. Supported by the male origin of mIMCD3 cells, $P K D 1 / 2>A R>C D H 2$ signaling in cluster $\mathrm{A}_{10}$ may contribute to this phenotype (Figs. $4 \mathrm{e}, 6 \mathrm{~d}$ and Table S9). The $A R$ gene in $\mathrm{TF}_{7}$ encodes the steroid-hormone activated androgen receptor, which regulates eukaryotic gene expression and affects cellular proliferation and differentiation in many target tissues ${ }^{63}$. Consistent with a putative input from PC-1/TRPP2, AR transcription factor activity is modulated by bound coactivator and corepressor proteins as well as post-translational modifications ${ }^{64}$. AR-regulated $C D H 2$ encodes cadherin-2, a $\mathrm{Ca}^{2+}$-dependent cell adhesion protein that is known to be important for the establishment and maintenance of the multicellular structure of organs ${ }^{65}$. Notably, renal proximal tubular cells are the only epithelial cells in the adult organism expressing cadherin-2 as major cell-cell adhesion protein ${ }^{66}$. Up-regulation of $\mathrm{CDH} 2$ is a hallmark of EMT, which has been described extensively for cyst-lining cells (Figure S6d) ${ }^{67,68}$. Further investigation of $P K D 1 / 2>A R>C D H 2$ may hence link several important disease-modulating factors in men with ADPKD.

Within the Epithelial Cell Differentiation cluster $\mathrm{B}_{8}, P K D 1 / 2>$ ETS $2>C D 34$ may provide another interesting model to test (Figs. 4e, 6d). ETS2 encodes the epithelium-specific, winged helix-turn-helix E26 transformationspecific proto-oncogene 2 transcription factor (ETS2) that regulates diverse cellular activities, including apoptosis, cell growth, adhesion, the extracellular matrix and other transcription factors ${ }^{69}$. ETS2 is a labile protein with a short half-life ${ }^{70}$. ETS2 transcription factor activity is regulated by E3 ligase-dependent ubiquitination and subsequent proteasomal degradation ${ }^{71,72}$. Interestingly, PC-1/TRPP2 has already been shown to regulate the activity of two E3 ligases, SIAH-1 and c-CBL ${ }^{73-75}$. Also, ETS2 is a downstream effector of the RAS/RAF/ERK pathway that promotes disease pathogenesis in $\mathrm{ADPKD}^{76}$. CD34 is regulated by ETS2 and is a common marker for diverse progenitor cells with progressive downregulation on more mature cells ${ }^{77}$. Functionally, CD34 is a sialomucin-type single-transmembrane glycophosphoprotein closely related to podocalyxin that seems to alter cellular adhesive properties ${ }^{78,79}$. CD34 is furthermore intracellularly coupled to CRKL, which has been associated with congenital kidney anomalies in DiGeorge Syndrome, including microcystic tubules and glomeruli ${ }^{80,81}$. The observed PKD-dependent over-expression of CD34 may therefore contribute to cystogenesis by causing cellular de-differentiation that interferes with renal tubular stability (Figure S6d).

In cluster $\mathrm{C}_{7}$ we identified CSF2RA, PIK3R1 and PLCB1 as novel genes responsive to PKD1 and PKD2, but not enough biobank information was available to automatically develop transcription-based regulatory signaling models (Figs. 4e, 6d). However, functional links to ADPKD have been described previously for PIK3R1 and PLCB1. PIK3R1 encodes phosphoinositide-3-kinase regulatory subunit 1 (PIK3R1), the major suppressive regulatory subunit of phosphoinositide 3-kinase (PI3K). PI3K participates in a broad range of regulatory processes, including cell growth, proliferation, metabolism and secretion ${ }^{82}$. In cancer, loss of PIK3R1 has been shown to promote EMT and cellular proliferation by over-activation of downstream AKT signaling ${ }^{83}$. In ADPKD, increased PI3K/AKT signaling has been described as disease modifying, but both, loss and gain of PKD1 have been linked to mis-regulated pathway activity ${ }^{84-88}$. Increased PIK3R1 protein expression has been observed in conditionally immortalized human tubular epithelial cells from ADPKD patients ${ }^{89}$. Disease status, species differences or the observed Pkd-dependent Pik3r1 exon usage may reconcile these divergent findings, but additional experimental data are needed to clarify the molecular function of PIK3R1 in ADPKD. PLCB1 encodes phospholipase C beta 1 (PLC $\beta 1$ ), which catalyzes the formation of inositol 1,4,5-trisphosphate $\left(\mathrm{PiP}_{3}\right)$ and diacylglycerol (DAG) from phosphatidylinositol 4,5-bisphosphate $\left(\mathrm{PiP}_{2}\right)$. PLC $\beta 1$ uses $\mathrm{Ca}^{2+}$ as a cofactor and plays an important role in the intracellular transduction of many extracellular signals ${ }^{90} . \mathrm{Ga}_{\mathrm{q}}$-mediated activation of PLC has been described downstream of PC-191.

Our transcriptomic analyses yielded novel, testable hypotheses and highlighted our still rather limited understanding of cellular physiology. Publicly available biobank data allowed for the identification and cross-correlation of $\mathrm{ABC}_{25}$, but were insufficient to develop unbiased hypotheses for most variations in $\mathrm{PKD}$-dependent gene expression. To date, combination of sophisticated model systems and high-resolution methods yield volumes of relevant transcriptomic data. Yet, interpretation has proven challenging when it comes to deriving biological meaning, as most types of systemic analysis, such as over-representation analysis, GSEA and signaling pathway impact analysis, rely to some degree on a priori knowledge of the pathways, the biological role, or the molecular function of genes ${ }^{38,92}$. As such, annotations have been instrumental for understanding molecular signatures of many diseases. But annotation-based approaches have significant drawbacks that limit the insights that can be gleaned from respective analyses. For instance, there is a strong underlying assumption that orthologous genes share similar biological functions. This may overemphasize highly conserved cellular processes and potentially overlook important species-specific and/or tissue-specific functions ${ }^{93}$. Similar network effects cause well-studied genes to continuously accrue data, while the non-randomness of missing annotations is more likely to disadvantage primate specific genes or new targets without a wealth of literature ${ }^{94,95}$. With more data becoming publicly available, future in silico re-analyses of $\mathrm{CD}_{178}$ have therefore the intriguing potential to further clarify core functional connections currently still hidden in the ADPKD transcriptomic profile.

Taken together, our data indicate the robust power of integrative transcriptomics to select targets for subsequent functional studies in ADPKD, including the 6 replicated (C3, FSTL1, PCOLCE, PCSK9, SPP1 and ZFP37) and 8 newly identified (CD34, CDH2, CSF2RA, DLX5, HOXC9, PIK3R1, PLCB1 and TLR6) genes (Fig. 6d). 


\section{Methods}

Contact for reagent and resource sharing. Further information and requests for resources should be directed to the Lead Contacts, Michael Köttgen (michael.koettgen@uniklinik-freiburg.de) and Alexis Hofherr (alexis.hofherr@uniklinik-freiburg.de).

Key resources. Details on key resources are listed in the supplementary information Key Resources Table.

Experimental model details. Cell culture. Murine inner-medullary collecting duct cells (mIMCD3) were isolated from healthy mice expressing the early region of simian virus SV40 (Tg(SV40E)Bri/7) to sustain continuous proliferation in vitro ${ }^{22}$. Wild-type mIMCD3 cells are diploid, consistently differentiate and replicate collecting duct-like transport activity ${ }^{22-25} . P k d 1^{-1-}$ and $P k d 2^{-1-}$ mIMCD3 cells have been described previously ${ }^{28}$. Independent deletion of $P k d 1$ (CRISPR) and $P k d 2$ (TALEN) was confirmed by DNA sequencing and validated by RNA-Seq: mIMCD3 $P k d 1^{-1-}(\Delta$-chr. 17: 24,550,055-24,594,963/ $\Delta$-chr. 17: 24,550,106-24,594,903); mIMCD3 $P k d 2^{-1-}\left(\Delta\right.$-chr. 5: 104,478,281-104,490,878) ${ }^{28}$. RNA-seq read mapping and de novo transcriptome assembly did not indicate significant genome-editing induced off-target effects in $P k d 1 / 2$ cells. Flow cytometry-based DNA content measurement showed normal ploidy in all cell lines. Loss of respective PC-1 and TRPP2 expression was demonstrated by Western blot. Overall viability, proliferation and differentiation, including ciliation, basal lamina formation and transepithelial electrical resistance, were similar in wild-type and mutant cells ${ }^{28}$.

Cells were cultured in a mix of Dulbecco's modified eagle's and F12 medium (DMEM-F12; Lonza), which was supplemented with $10 \%$ fetal bovine serum and $1 \%$ Penicillin-Streptomycin (10,000 U Penicillin and $10 \mathrm{mg} / \mathrm{ml}$ Streptomycin; both Sigma Aldrich). Cells were grown at $37^{\circ} \mathrm{C}$ and $10 \% \mathrm{CO}_{2}$. PCR-based testing for mycoplasma contamination was performed regularly. New ampules were started after $\sim 25$ passages. Cells were harvested using $0.25 \%$ Trypsin-EDTA (Gibco). Cell counts were performed by Countess II cell counter (Thermo Fisher).

Method details. Protein isolation, SDS-PAGE, Western blot and ECL detection. Cells were grown to epithelial confluency (5 days). Cells were lysed, proteins were isolated, processed in SDS-PAGE and Western blot and detected by electrochemiluminescence as described previously ${ }^{101}$. For TRPP2-immunoprecipitation, mouse anti-TRPP2 antibody was used (Santa Cruz Biotechnologie Inc., Dallas, USA). Beta-actin was targeted using anti-beta-actin antibody (Sigma Aldrich, St. Louis, USA). Luminescence was detected by a 16-bit ChemoCam system (Intas).

Immunofluorescence. Indirect immunofluorescence staining of cells has been described previously ${ }^{102}$. Briefly, after reaching full confluency cells were permeabilized using Triton X-100 (Carl Roth GmbH, Karlsruhe, Germany) and fixed by paraformaldehyde (Electron Microscopy Sciences, Hatfield, PA, USA). Cells were stained by primary (1:50 anti-ZO1 rat, Santa Cruz Biotechnology, Dallas, USA, 1:800; anti-alphaTubulin rabbit, Sigma Aldrich, St. Louis, USA) and secondary antibodies (1:1000 Anti-rat IgG, Cy3, Jackson Lab. Inc., Waltham, USA; anti-rabbit IgG, Cy3; Jackson Lab. Inc., Waltham, USA) in PBS. DAPI (Sigma-Aldrich) was added in a dilution of 1:1000 to visualize DNA. Bright-field images were recorded using an Axio Vert Observer microscope Z1 (Zeiss).

RNA isolation. For wildtype $/ \mathrm{Pkd}$ comparison, six $10 \mathrm{~cm}$ cell culture dishes were grown per genotype to full confluency and ciliation, starting with $5 \times 10^{6}$ cells per dish. RNA Isolation was performed 4 days after full confluency according to manufacturer's protocol using the RNeasy Plus Mini Kit (Qiagen), harvesting $2 \times 10^{6}$ cells per dish. Purification of RNA samples was performed by solvent extraction with $100 \%$ ethanol followed by precipitation with ammonium acetate. $10 \mu \mathrm{l}$ glycogen was added to $100 \mu \mathrm{l}$ RNA solution. RNA was solved in $10 \mu \mathrm{l}$ ammonium acetate $(7.5 \mathrm{M})$ and $250 \mu \mathrm{l}$ ethanol $(100 \%)$. After $30 \mathrm{~min}$ at $-80^{\circ} \mathrm{C}$ RNA was precipitated and separated by centrifugation for $30 \mathrm{~min}$ with $13,000 \mathrm{rpm}$ at $4{ }^{\circ} \mathrm{C}$. RNA was then washed twice in $200 \mu \mathrm{l}$ cold ethanol (80\%) followed by $30 \mathrm{~min}$ centrifugation with $13,000 \mathrm{rpm}$ at $4{ }^{\circ} \mathrm{C}$. RNA pellets were dried for $5 \mathrm{~min}$ at room temperature and resuspended in 10-15 $\mu \mathrm{l}$ RNAse-free water. Quality of RNA was checked by a Nanodrop photometer (Thermo Fisher). Four of the six samples were chosen for RNA-seq according to highest ratios for A260/280 (>2.0) and A260/230 (>2.0). RNA-seq was performed by GATC (Konstanz, D) on Illumina HiSEQ2500.

RNA sequencing (RNA-seq) analysis. RNA-seq was performed in samples from wild-type as well as from one $P k d 1^{-1-}$ and one $P k d 2^{-1-}$ mIMCD3 clone. Despite multiple sampling, untargeted analysis of such clonal cell lines may be confounded by undetected, random genome editing-induced off-target effects or clonal artifacts. To minimize false discovery, mIMCD3-based transcriptomic profiling of ADPKD was hence restricted to consistent changes across the two independent Pkd genotypes. Data was analyzed with Galaxy Europe ${ }^{103}$ (https:// usegalaxy.eu/). Reads were first trimmed and quality checked with Trim Galore (Galaxy Tool Version 0.4.3.1) and FastQC (Galaxy Tool Version 0.72), respectively. Reads were aligned to the mouse genome version mm10 $\left(\right.$ GRCm38) applying HISAT2 (Galaxy Tool Version 2.1.0 + galaxy3) ${ }^{104}$. Mapped reads were counted with htseqcount (Galaxy Tool Version 0.9.1) ${ }^{105}$. Differential gene expression (DGE) was tested using DESeq2 (Galaxy Tool Version 2.11.40.4) using a cutoff of $\log _{2}$ fold change of $\geq 1$ for upregulated and $\leq-1$ for downregulated genes ${ }^{35,106}$. False discovery rate of DGE was controlled with a p value $<0.05$ adjusted for multiple testing (Benjamini-Hochberg procedure). Principal Component Analysis (PCA) was performed using DESeq 2 in $\mathrm{R}^{106}$. Of $25^{\prime} 061$ genes with reads obtained in the RNA-seq analysis, $10 \%$ most variant genes were selected as input for PCA $(\mathrm{n}=2506)$. Data was visualized using the R packages: pcaExplorer and pheatmap ${ }^{107}$. 
Alternative splicing analysis. The reads processed in the RNA-seq analysis were also aligned to the reference genome and tailored for StringTie tool with HISAT2 (Galaxy Tool Version 2.1.0 + galaxy3) ${ }^{104}$. De novo transcript reconstruction was conducted with StringTie (Galaxy Tool Version 1.3.6) ${ }^{108}$. The transcriptome was assembled using StringTie-merge (Galaxy Tool Version 1.3.6). Assembled transcripts were compared to the reference annotation (Mus musculus, mm9) with GFFCompare (Galaxy Tool Version 0.11.2) ${ }^{109}$. Exon abundancies were then estimated using DEXSeq-Count (Galaxy Tool Version 1.28.1.0 $)^{99,100}$. Differential exon usage (DEU) was tested with DEXSeq (Galaxy Tool Version $1.28 .1+$ galaxy1) ${ }^{99,100}$. False discovery rate of DEU was controlled with a p-value $<0.05$ adjusted for multiple testing (Benjamini-Hochberg procedure).

CellNet analysis. CellNet Analysis of RNA-seq data was performed as described previously ${ }^{27}$. Data was uploaded to an Amazon web services (AWS) instance. No alterations to CellNet standard settings were performed. For the original code to work with the chosen AWS platform, all AWS-related URLs (https://s3.amazo naws.com/) were changed to https://s3.console.aws.amazon.com/s3/, e.g. in the "cn_s3_fetchFastq" function.

Kidney cell explorer analysis. Kidney Cell Explorer Analysis was performed using ${ }^{47}$ https://cello.shinyapps.io/ kidneycellexplorer/. "Adult mouse kidney" was selected as reference data set. $\mathrm{CD}_{178}$ were used as input by pasting the respective gene IDs in the search field. Expression was displayed in "Average expression (rescaled)" mode and "RdYIBu" Palette. Genes were counted as "expressed" if rescaled average expression was $>0$, e.g. if color was lighter than dark blue (HEX code \#4575b4ff.).

Nephro cell analysis. Nephro cell analysis was performed using http://nephrocell.miktmc.org/. "Adult normal kidney" was selected as reference data set. Rep $\mathrm{p}_{31}$ and $\mathrm{New}_{61}$ were used as input; one gene at a time. Violin plots displayed were analyzed and divided into 5 categories: $0=$ not measured in the nephrocell data set; $1=$ no expression; 2 = low expression, no violin plots displayed; 3 = high expression, violin plots displayed; $4=$ very high expression, striking violin plots displayed. A heatmap was generated to visualize these values.

Transcription factor enrichment analysis. Testing for transcription factors regulating the core data set genes was performed with ShinyGO (Tool Version 0.51) using the function TF.Target.TFacts ${ }^{41,42}$. False discovery rate was set to $<0.05$.

Pathway analysis. Functional protein network and transcriptional factor network were determined using the STRING (Search tool for the retrieval of interacting genes/proteins) database version $11.0^{37}$ (https://version11-0.string-db.org/).

Functional enrichment analysis was performed against the Molecular Signature Database (MSigDB) v7.1 using standard hypergeometric distribution with correction for multiple hypotheses testing using FDR $<0.05$ according to Benjamini and Hochberg ${ }^{38,42}$. Pathway databases used: Hallmark Gene Sets, Positional Gene Sets, GO Gene Sets, KEGG, All Transcription Factor Targets.

Cross-species meta-analysis. Available PKD1-responsive transcriptional data was retrieved from a literature research covering publications between 2008 and $2018^{12-17}$. Transgenic models were not considered for comparison. Studies were included if they provided lists of differentially expressed genes and raw data had been deposited for public access. No PKD2 data matching above criteria were found. Ref. ${ }^{18}$ was included for PKD2responsive expression. Lists of differentially expressed genes were obtained from the primary or supplementary data of the respective publications. Analysis of ${ }^{13,16}$ was based on the previously described PKD1 Signature by ${ }^{15}$. Criteria for differential gene expression were adopted from the respective studies.

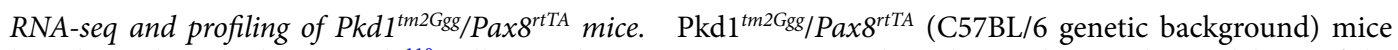
have been described previously ${ }^{110}$. All animal experiments were conducted according to the guidelines of the National Institutes of Health Guide for the Care and Use of Laboratory Animals, as well as the German law for the welfare of animals, and were approved by regional authorities (Regierungspräsidium Freiburg G-13/18, G-15/58, and G-16/28). Mice were housed in a specific pathogen-free facility, fed ad libitum, and housed at constant ambient temperature in a 12-hour day/night cycle. Breeding and genotyping were done according to standard procedures. $P k d 1$ deletion was induced from post-natal day $28-42$. Mice received doxycycline hydrochloride (Fagron) via the drinking water $(2 \mathrm{mg} / \mathrm{mL}$ with $5 \%$ sucrose, protected from light) to induce Pkd1 deletion. Littermates (lacking either TetO-Cre or Pax $8^{\text {rtTA }}$ ) were used as controls. 2 male control animals and 5 male $P k d 1^{-/-}$ animals were sacrificed at a median age of 11.8 weeks. RNA was extracted from kidneys with RNeasy Plus Mini Kit (Qiagen) according to the manufacturer's protocol as a one-step elution with $50 \mu$ l RNAse free water per sample. Quantity and quality of RNA was checked by NanoDrop One spectrophotometer (Thermofisher). RNAseq was performed by GATC Biotech AG (Konstanz, D) on Illumina HiSeq Genome Sequencer. Data analysis was performed using the same work-flow as for $\mathrm{CD}_{178}$ (see "RNA Sequencing analysis"). Differentially expressed genes $(\mathrm{FDR}<0.05)$ were overlapped to top $20 \%$ genes contributing to principal component 2 in the principal component analysis. Overlapping genes were summarized in the $P k d 1^{-/-}$mouse data set.

Quantification and statistical analysis. Statistical analyses of RNA-seq data were carried out in Galaxy and $\mathrm{R}$ using DESeq $2^{103,106}$. False discovery rate of DGE was set to $<0.05$ (Benjamini-Hochberg procedure). The number of biological replicates (n), statistical test performed and resulting significance for each experiment is 
noted in the figure legend or the corresponding results section of this document. Where applicable, original data for plots is available in supplementary tables. Additional data is available upon request.

\section{Data availability}

The raw and processed data from our analyses of RNA-seq will be available in the gene expression omnibus database under the accession number: GSE179947.

Received: 17 March 2021; Accepted: 8 July 2021

Published online: 23 July 2021

\section{References}

1. Cornec-Le Gall, E., Alam, A. \& Perrone, R. D. Autosomal dominant polycystic kidney disease. Lancet 393(10174), 919-935 (2019).

2. Mochizuki, T. et al. PKD2, a gene for polycystic kidney disease that encodes an integral membrane protein. Science 272(5266), 1339-1342 (1996).

3. Polycystic kidney disease: the complete structure of the PKD1 gene and its protein. The International Polycystic Kidney Disease Consortium. Cell 81(2), 289-298 (1995).

4. Shillingford, J. M. et al. The mTOR pathway is regulated by polycystin-1, and its inhibition reverses renal cystogenesis in polycystic kidney disease. Proc. Natl. Acad. Sci. USA. 103(14), 5466-5471 (2006).

5. Kim, S. et al. The polycystin complex mediates Wnt/Ca(2+) signalling. Nat. Cell Biol. 18(7), 752-764 (2016).

6. Happe, H. et al. Altered Hippo signalling in polycystic kidney disease. J. Pathol. 224(1), 133-142 (2011).

7. Bhunia, A. K. et al. PKD1 induces 21 (waf1) and regulation of the cell cycle via direct activation of the JAK-STAT signaling pathway in a process requiring PKD2. Cell 109(2), 157-168 (2002).

8. Hofherr, A. et al. The mitochondrial transporter SLC25A25 links ciliary TRPP2 signaling and cellular metabolism. PLoS Biol. 16(8), e2005651 (2018).

9. Antignac, C. et al. The future of polycystic kidney disease research-as seen by the 12 Kaplan Awardees. J. Am. Soc. Nephrol. 26(9), 2081-2095 (2015).

10. Wang, Z., Gerstein, M. \& Snyder, M. RNA-Seq: a revolutionary tool for transcriptomics. Nat. Rev. Genet. 10(1), 57-63 (2009).

11. Kim, H. \& Kim, J. S. A guide to genome engineering with programmable nucleases. Nat. Rev. Genet. 15(5), 321-334 (2014).

12. Kunnen, S. J. et al. Comparative transcriptomics of shear stress treated Pkd1 $(-/-)$ cells and pre-cystic kidneys reveals pathways involved in early polycystic kidney disease. Biomed. Pharmacother. 108, 1123-1134 (2018).

13. Menezes, L. F. et al. Network analysis of a Pkd1-mouse model of autosomal dominant polycystic kidney disease identifies HNF4alpha as a disease modifier. PLoS Genet. 8(11), e1003053 (2012).

14. Chen, W. C., Tzeng, Y. S. \& Li, H. Gene expression in early and progression phases of autosomal dominant polycystic kidney disease. BMC Res. Notes. 1, 131 (2008).

15. Malas, T. B. et al. Meta-analysis of polycystic kidney disease expression profiles defines strong involvement of injury repair processes. Am. J. Physiol. Renal. Physiol. 312(4), F806-F817 (2017).

16. Song, X. et al. Systems biology of autosomal dominant polycystic kidney disease (ADPKD): computational identification of gene expression pathways and integrated regulatory networks. Hum. Mol. Genet. 18(13), 2328-2343 (2009).

17. Pandey, P., Qin, S., Ho, J., Zhou, J. \& Kreidberg, J. A. Systems biology approach to identify transcriptome reprogramming and candidate microRNA targets during the progression of polycystic kidney disease. BMC Syst. Biol. 5, 56 (2011).

18. Zhang, C. et al. Cyclin-dependent kinase 1 activity is a driver of cyst growth in polycystic kidney disease. J. Am. Soc. Nephrol. 32(1), 41-51 (2021).

19. Terabayashi, T., Germino, G. G. \& Menezes, L. F. Pathway identification through transcriptome analysis. Cell Signal. 74, 109701 (2020).

20. Qian, F., Watnick, T. J., Onuchic, L. F. \& Germino, G. G. The molecular basis of focal cyst formation in human autosomal dominant polycystic kidney disease type I. Cell 87(6), 979-987 (1996).

21. Formica, C. \& Peters, D. J. M. Molecular pathways involved in injury-repair and ADPKD progression. Cell Signal. 72, 109648 (2020).

22. Rauchman, M. I., Nigam, S. K., Delpire, E. \& Gullans, S. R. An osmotically tolerant inner medullary collecting duct cell line from an SV40 transgenic mouse. Am. J. Physiol. 265(3 Pt 2), F416-424 (1993).

23. Kaplan, M. R., Plotkin, M. D., Brown, D., Hebert, S. C. \& Delpire, E. Expression of the mouse Na-K-2Cl cotransporter, mBSC2, in the terminal inner medullary collecting duct, the glomerular and extraglomerular mesangium, and the glomerular afferent arteriole. J. Clin. Invest. 98(3), 723-730 (1996).

24. Amlal, H., Goel, A. \& Soleimani, M. Activation of $\mathrm{H}+$-ATPase by hypotonicity: a novel regulatory mechanism for $\mathrm{H}+$ secretion in IMCD cells. Am. J. Physiol. 275(4), F487-501 (1998).

25. Soleimani, M., Singh, G., Bizal, G. L., Gullans, S. R. \& McAteer, J. A. Na+/H+ exchanger isoforms NHE-2 and NHE-1 in inner medullary collecting duct cells: expression, functional localization, and differential regulation. J. Biol. Chem. 269(45), 27973-27978 (1994).

26. Aboudehen, K. et al. Long noncoding RNA Hoxb3os is dysregulated in autosomal dominant polycystic kidney disease and regulates mTOR signaling. J. Biol. Chem. 293(24), 9388-9398 (2018).

27. Radley, A. H. et al. Assessment of engineered cells using Cell Net and RNA-seq. Nat. Protoc. 12(5), 1089-1102 (2017).

28. Hofherr, A. et al. Efficient genome editing of differentiated renal epithelial cells. Pflugers Arch. 469(2), 303-311 (2017).

29. Elowitz, M. B., Levine, A. J., Siggia, E. D. \& Swain, P. S. Stochastic gene expression in a single cell. Science 297(5584), 1183-1186 (2002).

30. Churchill, G. A. Fundamentals of experimental design for cDNA microarrays. Nat. Genet. 32(Suppl), 490-495 (2002).

31. Hansen, K. D., Wu, Z., Irizarry, R. A. \& Leek, J. T. Sequencing technology does not eliminate biological variability. Nat. Biotechnol. 29(7), 572-573(2011).

32. Marioni, J. C., Mason, C. E., Mane, S. M., Stephens, M. \& Gilad, Y. RNA-seq: an assessment of technical reproducibility and comparison with gene expression arrays. Genome Res. 18(9), 1509-1517 (2008).

33. Kim, S., Kang, D., Huo, Z., Park, Y. \& Tseng, G. C. Meta-analytic principal component analysis in integrative omics application. Bioinformatics 34(8), 1321-1328 (2018).

34. Costa-Silva, J., Domingues, D. \& Lopes, F. M. RNA-Seq differential expression analysis: an extended review and a software tool. PLOS ONE 12(12), e0190152 (2017).

35. Consortium $\mathrm{M}$ et al. The MicroArray Quality Control (MAQC) project shows inter- and intraplatform reproducibility of gene expression measurements. Nat. Biotechnol. 24(9), 1151-1161 (2006).

36. Conesa, A. et al. A survey of best practices for RNA-seq data analysis. Genome Biol. 17, 13 (2016). 
37. Szklarczyk, D. et al. STRING v11: protein-protein association networks with increased coverage, supporting functional discovery in genome-wide experimental datasets. Nucleic Acids Res. 47(D1), D607-D613 (2019).

38. Subramanian, A. et al. Gene set enrichment analysis: a knowledge-based approach for interpreting genome-wide expression profiles. Proc. Natl. Acad. Sci. USA. 102(43), 15545-15550 (2005).

39. Schieren, G. et al. Gene profiling of polycystic kidneys. Nephrol. Dial Transplant. 21(7), 1816-1824 (2006).

40. Kanehisa, M., Sato, Y., Kawashima, M., Furumichi, M. \& Tanabe, M. KEGG as a reference resource for gene and protein annotation. Nucleic Acids Res. 44(D1), D457-462 (2016).

41. Ge, S. X., Jung, D. \& Yao, R. ShinyGO: a graphical gene-set enrichment tool for animals and plants. Bioinformatics 36(8), $2628-2629(2020)$.

42. Liberzon, A. et al. The molecular signatures database (MSigDB) hallmark gene set collection. Cell Syst. 1(6), 417-425 (2015).

43. Su, Q. et al. Structure of the human PKD1-PKD2 complex. Science 361, 6406 (2018).

44. Delling, M., DeCaen, P. G., Doerner, J. F., Febvay, S. \& Clapham, D. E. Primary cilia are specialized calcium signalling organelles. Nature 504(7479), 311-314 (2013).

45. Niewiadomski, P. et al. Gli protein activity is controlled by multisite phosphorylation in vertebrate Hedgehog signaling. Cell Rep. 6(1), 168-181 (2014).

46. Matlin, A. J., Clark, F. \& Smith, C. W. Understanding alternative splicing: towards a cellular code. Nat. Rev. Mol. Cell Biol. 6(5), 386-398 (2005).

47. Ransick, A. et al. Single-cell profiling reveals sex, lineage, and regional diversity in the mouse kidney. Dev. Cell. 51(3), 399-413. e397 (2019).

48. Han, Y., Gao, S., Muegge, K., Zhang, W. \& Zhou, B. Advanced applications of RNA sequencing and challenges. Bioinform. Biol. Insights. 9(Suppl 1), 29-46 (2015).

49. Menon, R. et al. Single cell transcriptomics identifies focal segmental glomerulosclerosis remission endothelial biomarker. JCI Insight. 5, 6 (2020).

50. Vollmann, E. H. et al. Identification of novel fibrosis modifiers by in vivo siRNA silencing. Mol. Ther. Nucleic Acids. 7, 314-323 (2017).

51. Anders, H. J., Banas, B. \& Schlondorff, D. Signaling danger: toll-like receptors and their potential roles in kidney disease. J. Am. Soc. Nephrol. 15(4), 854-867 (2004).

52. Claussnitzer, M. et al. A brief history of human disease genetics. Nature 577(7789), 179-189 (2020).

53. Desnick, R. J. \& Schuchman, E. H. Enzyme replacement and enhancement therapies: lessons from lysosomal disorders. Nat. Rev. Genet. 3(12), 954-966 (2002).

54. Finkel, R. S. et al. Treatment of infantile-onset spinal muscular atrophy with nusinersen: a phase 2, open-label, dose-escalation study. Lancet 388(10063), 3017-3026 (2016).

55. Atkin, T. A. et al. A comprehensive approach to identifying repurposed drugs to treat SCN8A epilepsy. Epilepsia 59(4), 802-813 (2018).

56. Liu, Y., Beyer, A. \& Aebersold, R. On the dependency of cellular protein levels on mRNA abundance. Cell 165(3), 535-550 (2016).

57. Fasterius, E. \& Al-Khalili, S. C. Analysis of public RNA-sequencing data reveals biological consequences of genetic heterogeneity in cell line populations. Sci. Rep. 8(1), 11226 (2018).

58. Nikhil, K. L., Korge, S. \& Kramer, A. Heritable gene expression variability and stochasticity govern clonal heterogeneity in circadian period. PLoS Biol. 18(8), 3000792 (2020).

59. Li, Y. et al. Noise-driven cellular heterogeneity in circadian periodicity. Proc. Natl. Acad. Sci. USA. 117(19), 10350-10356 (2020).

60. Rung, J. \& Brazma, A. Reuse of public genome-wide gene expression data. Nat. Rev. Genet. 14(2), 89-99 (2013).

61. Reed, B. Y. et al. Variation in age at ESRD in autosomal dominant polycystic kidney disease. Am. J. Kidney Dis. 51(2), 173-183 (2008).

62. Cnossen, W. R. \& Drenth, J. P. Polycystic liver disease: an overview of pathogenesis, clinical manifestations and management. Orphanet. J. Rare Dis. 9,69 (2014).

63. Bolton, E. C. et al. Cell- and gene-specific regulation of primary target genes by the androgen receptor. Genes Dev. 21(16), 2005-2017 (2007).

64. Culig, Z. Androgen receptor coactivators in regulation of growth and differentiation in prostate cancer. J. Cell Physiol. 231(2), 270-274 (2016).

65. Halbleib, J. M. \& Nelson, W. J. Cadherins in development: cell adhesion, sorting, and tissue morphogenesis. Genes Dev. 20(23), 3199-3214 (2006).

66. Nouwen, E. J., Dauwe, S., van der Biest, I. \& De Broe, M. E. Stage- and segment-specific expression of cell-adhesion molecules N-CAM, A-CAM, and L-CAM in the kidney. Kidney Int. 44(1), 147-158 (1993).

67. Kim, D. Y. et al. Impact of miR-192 and miR-194 on cyst enlargement through EMT in autosomal dominant polycystic kidney disease. FASEB J. 33(2), 2870-2884 (2019).

68. Roitbak, T. et al. A polycystin-1 multiprotein complex is disrupted in polycystic kidney disease cells. Mol. Biol. Cell. 15(3), $1334-1346$ (2004).

69. Findlay, V. J., LaRue, A. C., Turner, D. P., Watson, P. M. \& Watson, D. K. Understanding the role of ETS-mediated gene regulation in complex biological processes. Adv. Cancer Res. 119, 1-61 (2013).

70. Fujiwara, S., Fisher, R. J., Bhat, N. K., DiazdelaEspina, S. M. \& Papas, T. S. A short-lived nuclear phosphoprotein encoded by the human ets-2 proto-oncogene is stabilized by activation of protein kinase C. Mol. Cell Biol. 8(11), 4700-4706 (1988).

71. Li, M. et al. The adaptor protein of the anaphase promoting complex Cdh1 is essential in maintaining replicative lifespan and in learning and memory. Nat. Cell Biol. 10(9), 1083-1089 (2008).

72. Guen, V. J. et al. CDK10/cyclin M is a protein kinase that controls ETS2 degradation and is deficient in STAR syndrome. Proc. Natl. Acad. Sci. USA. 110(48), 19525-19530 (2013).

73. Foy, R. L. et al. Polycystin-1 regulates the stability and ubiquitination of transcription factor Jade-1. Hum. Mol. Genet. 21(26), 5456-5471 (2012).

74. Kim, H., Jeong, W., Ahn, K., Ahn, C. \& Kang, S. Siah-1 interacts with the intracellular region of polycystin-1 and affects its stability via the ubiquitin-proteasome pathway. J. Am. Soc. Nephrol. 15(8), 2042-2049 (2004).

75. Qin, S. et al. Failure to ubiquitinate c-Met leads to hyperactivation of mTOR signaling in a mouse model of autosomal dominant polycystic kidney disease. J. Clin. Invest. 120(10), 3617-3628 (2010).

76. Su, L. et al. Ganoderma triterpenes retard renal cyst development by downregulating Ras/MAPK signaling and promoting cell differentiation. Kidney Int. 92(6), 1404-1418 (2017).

77. Sidney, L. E., Branch, M. J., Dunphy, S. E., Dua, H. S. \& Hopkinson, A. Concise review: evidence for CD34 as a common marker for diverse progenitors. Stem Cells. 32(6), 1380-1389 (2014).

78. Melotti, P. \& Calabretta, B. Ets-2 and c-Myb act independently in regulating expression of the hematopoietic stem cell antigen CD34. J. Biol. Chem. 269(41), 25303-25309 (1994).

79. Nielsen, J. S. \& McNagny, K. M. Novel functions of the CD34 family. J. Cell Sci. 121(Pt 22), 3683-3692 (2008).

80. Lopez-Rivera, E. et al. Genetic drivers of kidney defects in the DiGeorge syndrome. N. Engl. J. Med. 376(8), 742-754 (2017). 
81. Felschow, D. M., McVeigh, M. L., Hoehn, G. T., Civin, C. I. \& Fackler, M. J. The adapter protein CrkL associates with CD34. Blood 97(12), 3768-3775 (2001).

82. Fruman, D. A. et al. The PI3K pathway in human disease. Cell 170(4), 605-635 (2017).

83. Lin, Y. et al. PIK3R1 negatively regulates the epithelial-mesenchymal transition and stem-like phenotype of renal cancer cells through the AKT/GSK3beta/CTNNB1 signaling pathway. Sci. Rep. 5, 8997 (2015).

84. Boca, M. et al. Polycystin-1 induces resistance to apoptosis through the phosphatidylinositol 3-kinase/Akt signaling pathway. J. Am. Soc. Nephrol. 17(3), 637-647 (2006).

85. Gargalionis, A. N. et al. Polycystin-1 induces activation of the PI3K/AKT/mTOR pathway and promotes angiogenesis in renal cell carcinoma. Cancer Lett. 489, 135-143 (2020).

86. Boca, M. et al. Polycystin-1 induces cell migration by regulating phosphatidylinositol 3-kinase-dependent cytoskeletal rearrangements and GSK3beta-dependent cell cell mechanical adhesion. Mol. Biol. Cell. 18(10), 4050-4061 (2007).

87. Parker, E. et al. Hyperproliferation of PKD1 cystic cells is induced by insulin-like growth factor-1 activation of the Ras/Raf signalling system. Kidney Int. 72(2), 157-165 (2007).

88. Margaria, J. P., Campa, C. C., De Santis, M. C., Hirsch, E. \& Franco, I. The PI3K/Akt/mTOR pathway in polycystic kidney disease: a complex interaction with polycystins and primary cilium. Cell Signal. 66, 109468 (2020).

89. Magayr, T. A. et al. Global microRNA profiling in human urinary exosomes reveals novel disease biomarkers and cellular pathways for autosomal dominant polycystic kidney disease. Kidney Int. 98(2), 420-435 (2020).

90. Bill, C. A. \& Vines, C. M. Phospholipase C. Adv. Exp. Med. Biol. 1131, 215-242 (2020).

91. Puri, S. et al. Polycystin-1 activates the calcineurin/NFAT (nuclear factor of activated T-cells) signaling pathway. J. Biol. Chem. 279(53), 55455-55464 (2004).

92. Tarca, A. L. et al. A novel signaling pathway impact analysis. Bioinformatics 25(1), 75-82 (2009).

93. Gabaldon, T. \& Koonin, E. V. Functional and evolutionary implications of gene orthology. Nat. Rev. Genet. 14(5), 360-366 (2013).

94. Maertens, A. et al. Functionally enigmatic genes in cancer: using TCGA data to map the limitations of annotations. Sci. Rep. 10(1), $4106(2020)$.

95. Pandey, A. K., Lu, L., Wang, X., Homayouni, R. \& Williams, R. W. Functionally enigmatic genes: a case study of the brain ignorome. PLoS ONE 9(2), e88889 (2014).

96. Ashburner, M. et al. Gene ontology: tool for the unification of biology. The gene ontology consortium. Nat. Genet. 25(1), 25-29 (2000).

97. Gene, O. C. The Gene Ontology resource: enriching a GOld mine. Nucleic Acids Res. 49(D1), D325-D334 (2021).

98. Yevshin, I., Sharipov, R., Kolmykov, S., Kondrakhin, Y. \& Kolpakov, F. GTRD: a database on gene transcription regulation-2019 update. Nucleic Acids Res. 47(D1), D100-D105 (2019).

99. Anders, S., Reyes, A. \& Huber, W. Detecting differential usage of exons from RNA-seq data. Genome Res. 22(10), 2008-2017 (2012).

100. Reyes, A. et al. Drift and conservation of differential exon usage across tissues in primate species. Proc. Natl. Acad. Sci. USA. 110(38), 15377-15382 (2013).

101. Hofherr, A., Wagner, C. J., Watnick, T. \& Kottgen, M. Targeted rescue of a polycystic kidney disease mutation by lysosomal inhibition. Kidney Int. 89(4), 949-955 (2016).

102. Hofherr, A., Wagner, C., Fedeles, S., Somlo, S. \& Kottgen, M. N-glycosylation determines the abundance of the transient receptor potential channel TRPP2. J. Biol. Chem. 289(21), 14854-14867 (2014).

103. Afgan, E. et al. The Galaxy platform for accessible, reproducible and collaborative biomedical analyses: 2018 update. Nucleic Acids Res. 46(W1), W537-W544 (2018).

104. Kim, D., Paggi, J. M., Park, C., Bennett, C. \& Salzberg, S. L. Graph-based genome alignment and genotyping with HISAT2 and HISAT-genotype. Nat. Biotechnol. 37(8), 907-915 (2019).

105. Anders, S., Pyl, P. T. \& Huber, W. HTSeq-a Python framework to work with high-throughput sequencing data. Bioinformatics 31(2), 166-169 (2015).

106. Love, M. I., Huber, W. \& Anders, S. Moderated estimation of fold change and dispersion for RNA-seq data with DESeq2. Genome Biol. 15(12), 550 (2014).

107. Marini, F. \& Binder, H. pcaExplorer: an R/Bioconductor package for interacting with RNA-seq principal components. BMC Bioinform. 20(1), 331 (2019).

108. Pertea, M., Kim, D., Pertea, G. M., Leek, J. T. \& Salzberg, S. L. Transcript-level expression analysis of RNA-seq experiments with HISAT StringTie and Ballgown. Nat. Protoc. 11(9), 1650-1667 (2016).

109. Pertea, G., \& Pertea, M. GFF utilities: GffRead and GffCompare. F1000Res 9 (2020).

110. Viau, A. et al. Cilia-localized LKB1 regulates chemokine signaling, macrophage recruitment, and tissue homeostasis in the kidney. EMBO J. 37, 15 (2018).

\section{Acknowledgements}

We would like to thank Simone Diederichsen and Lukas Peintner for technical assistance and Anna Reznichenko for her input on the manuscript. The following illustrations for figure design were obtained from the DBCLS Togo TV picture gallery: mouse (Figures 1, 6, S5, S6); human (Figure 1, 6, S6); and cell culture (Figure 1, 6, S6).

\section{Author contributions}

A.H., M.K. and E.W.K. designed the research project. S.F., H.M., C.R., H.S., K.F., C.L.W., T.B. and A.V. and A.H. performed the experiments and acquired the data. A.H., S.F., H.M. and C.R.. analyzed the data. S.F., H.M., C.R., M.K. and A.H. drafted and revised the manuscript. All authors approved the final version of the manuscript.

\section{Funding}

Open Access funding enabled and organized by Projekt DEAL. M.K. is supported by the German Research Foundation (DFG): TRR 152 (project ID 239283807); SFB1453 (Project ID 431984000) and Germany's Excellence Strategy (CIBSS-EXC-2189-Project ID 390939984). E.W.K. is supported by the German Research Foundation (DFG): KU1504/7-1 and KU1504/8-1.

\section{Competing interests}

The authors declare no competing interests. 


\section{Additional information}

Supplementary Information The online version contains supplementary material available at https://doi.org/ 10.1038/s41598-021-94442-8.

Correspondence and requests for materials should be addressed to M.K. or A.H.

Reprints and permissions information is available at www.nature.com/reprints.

Publisher's note Springer Nature remains neutral with regard to jurisdictional claims in published maps and institutional affiliations.

(c) (i) Open Access This article is licensed under a Creative Commons Attribution 4.0 International License, which permits use, sharing, adaptation, distribution and reproduction in any medium or format, as long as you give appropriate credit to the original author(s) and the source, provide a link to the Creative Commons licence, and indicate if changes were made. The images or other third party material in this article are included in the article's Creative Commons licence, unless indicated otherwise in a credit line to the material. If material is not included in the article's Creative Commons licence and your intended use is not permitted by statutory regulation or exceeds the permitted use, you will need to obtain permission directly from the copyright holder. To view a copy of this licence, visit http://creativecommons.org/licenses/by/4.0/.

(C) The Author(s) 2021 